\title{
State and socio-demographic group variation in out-of-pocket expenditure, borrowings and Janani Suraksha Yojana (JSY) programme use for birth deliveries in India
}

\author{
Hanimi Reddy Modugu ${ }^{1 *}$, Manish Kumar², Ashok Kumar $^{3}$ and Christopher Millett ${ }^{4}$
}

\begin{abstract}
Background: High out-of-pocket-expenditure (OOPE) deters families from seeking skilled/institutional care. 'Janani Suraksha Yojana (JSY), a conditional cash transfer programme launched in 2005 to mitigate OOPE and to promote institutional deliveries among the poor, is part of Government of India's efforts to achieve Millennium Development Goals (MDGs) 4 and 5. The objective of this study is to estimate variations in OOPE for normal/caesarean-section deliveries, JSY-programme use and delivery associated borrowings - by states and union territories, and socio-demographic profiling of families, in India.

Methods: Secondary analysis of data from the District Level Household Survey (DLHS-3), 2007-08. Mean and median OOPE, percentage use of JSY and percentage of families needing to borrow money to pay for delivery associated expenditure was estimated for institutional and home deliveries.

Results: Half (52\%) of all deliveries in India occurred at home in 2007/08. OOPE for women having institutional deliveries remained high, with considerable variation between states and union territories. Mean OOPE (SD) of a normal delivery in public and private institution respectively in India were Rs. 1,624 and Rs. 4,458 and for a caesarean-section it was Rs. 5,935 and Rs. 14,276 respectively. There was considerable state-level variation in use of the JSY programme for normal deliveries (15\% nationally; ranging from 0\% in Goa to 43\% in Madhya Pradesh) and the percentage of families having to borrow money to pay for a caesarean-section in a private institution (47\% nationally; ranging from $7 \%$ in Goa to $69 \%$ in Bihar). Increased literacy and wealth were associated with a higher likelihood of an institutional delivery, higher OOPE but no major variations in use of the JSY.
\end{abstract}

Conclusions: Our study highlights the ongoing high OOPE and impoverishing impact of institutional care for deliveries in India. Supporting families in financial planning for maternity care, additional investment in the JSY programme and strengthening state level planning are required to increase the proportion of institutional deliveries.

\section{Background}

In India, high out-of-pocket expenditure (OOPE) is one of the main deterrents to seeking skilled/institutional care $[1,2]$. With OOPE on health increasing as a proportion of household expenditure [3], poor families (particularly the two lowest quintiles) are becoming particularly vulnerable when these expenditures exceed their capacity to pay [4].

\footnotetext{
* Correspondence: hanimi.reddy@phfi.org

'South Asia Network for Chronic Disease (SANCD), Public Health Foundation of India (PHFI), C1/52, First Floor, Safdarjung Development Area, New Delhi 110016, India

Full list of author information is available at the end of the article
}

In 2004-05, about 39 million Indians (around 4\% of population) fell into poverty due to OOPEs on health care $[5,6]$, including maternal health care.

Since maternal mortality is generally lower where a higher proportion of deliveries are conducted by skilled birth attendants, experts feel this should be a central element of any policy or programme that aims to reduce maternal deaths [7]. The maternal mortality ratio (MMR) in India declined substantially from 398 per 100,000 live births in 1997-98 [8] to 212 per 100,000 live births in 2007-09 [9], with under-five mortality rate (U5MR) declining from 109 per 1,000 live births in

\section{Biomed Central}


1992-93 [10] to 74 per 1,000 live births in 2005-06 [11]. The proportion of institutional deliveries increased from $39 \%$ in 2005-06 [11] to $73 \%$ in 2009 [12]. However, India is still far from achieving its Millennium Development Goals (MDGs) 4 and 5 (38 deaths per 1,000 live births for child mortality and less than 100 deaths per 100,000 births for maternal mortality) and universal institutional delivery care, by 2015 [13].

In 2005, Government of India launched 'Janani Suraksha Yojana (JSY)' programme', a safe motherhood intervention under the National Rural Health Mission (NRHM), with the objective of reducing maternal and neo-natal mortality by promoting institutional deliveries among the poor $[13,14]$. JSY is the largest conditional cash transfer programme in the world in terms of number of beneficiaries and constitutes a major Indian health care programme $[13,15]$. It is a centrally sponsored demand generation programme for 100 percent cash transfer to incentivise women/family to give birth in health facilities. Even though JSY is a centrally sponsored scheme, its implementation differs across the states and union territories [14]. Within five years JSY has made substantial strides, with the number of beneficiaries increasing from 0.74 million in 2005-06 to 10 million in 2009-10 [16], thus covering around 40 percent of total deliveries in the country. Its budgetary allocation has also increased from US\$ 8.5 million in 2005-06 to US\$ 275 million in 2008-09 [13].

\section{Janani Suraksha Yojana (JSY) programme guidelines}

According to JSY's guideline, after delivery in a public or accredited private health facility, eligible women receive $R s 600$ in urban areas and Rs 700 in rural areas. In ten High Focus- Non North Eastern (NE) states (Uttar Pradesh, Uttarakhand, Bihar, Jharkhand, Madhya Pradesh, Chhattisgarh, Himachal Pradesh, Rajasthan, Orissa, and Jammu E Kashmir) all pregnant women are eligible, and benefits are paid regardless of whether they deliver in a government or in a private accredited institution, and regardless of birth order. Benefits for institutional delivery are Rs. 1,400 in rural areas and Rs. 1,000 in urban areas. In Non High Focus states, women are eligible for the cash benefit only for their first two live births and only if they had a below poverty line (BPL) card issued by the government or if they were from a scheduled caste or tribe. Pregnant women can also receive cash assistance for transport to the nearest government health facility for delivery. Each state determines the amount of assistance, but the minimum is Rs. 250. It is paid to pregnant women on arrival and registration at the facility. Women who deliver at home are still eligible for a cash payment to cover the expenses associated with delivery, but only if they are 19 years of age and older, belong to BPL household and gave birth to their first or second child. Such mothers are entitled to Rs. 500 per delivery. JSY is being implemented through Accredited Social Health Activists (ASHAs), who identify pregnant women and help them to get to a health facility. ASHAs receive payments of Rs. 200 in urban areas and Rs. 600 in rural areas per in-facility delivery assisted by them in high focus states. $[14,15,17]$.

Although a small number of micro studies [18-22] have provided estimates of OOPE to family for delivery care, these estimates were confined to small geographic areas in India. We used a nationally representative cross-sectional dataset [District Level Household and Facility SurveyPhase 3 (DLHS-3)], to provide robust estimates of OOPE to family of delivery care for all the states and union territories in India, except for Nagaland, as it was not covered under DLHS-3. Specific objectives of our study are:

1. To estimate the average OOPE for women/families according to the type (normal/caesarean-section) and place (home/government hospital/private hospital) of delivery, in the states/union territories of India;

2. To examine inter-state variations in percent JSY beneficiaries and percent families who had to borrow money/sell property to meet the delivery expenses for normal and caesarean-section deliveries;

3. To outline how average OOPEs, percent JSY beneficiaries and percent families borrowing vary for normal/caesarean-section deliveries according to socio-demographic profiling of families in India.

\section{Methods}

The DLHS-3 collected data on OOPE to family on delivery care from ever married women who had a live/still birth between January 2004 and December 2008. However, we confined our analysis to births/deliveries between January 2007 and December 2008, as state-wise implementation of the JSY programme was highly variable during previous years [14].

We have adopted the DLHS-3 definition for 'type of delivery' and 'place of delivery' [23]. A delivery not requiring intervention in the form of an operation/use of forceps/ cut and stitches was termed 'normal vaginal delivery'; an operation was termed 'caesarean-section' ('c-section'); and the use of forceps/cut/ stitches was termed 'instrument/assisted' delivery. A delivery in a public institution [Government hospital, dispensary, urban health centre/post/family welfare centre, community health centre/rural hospital, primary health centre, sub centre, Ayurveda, Yoga, Unani, Siddha, \& Homeopathy (AYUSH) hospital/clinic] was classified as 'public 
institution delivery'. A delivery in a private hospital/clinic or private AYUSH hospital/clinic, was classified 'private institution delivery'. A delivery in a woman's or her parents' home was classified as 'home delivery', and a delivery occurring at a Non-governmental organisation (NGO)/Trust hospital/clinic, en route to the hospital, work place, other places was classified as 'other place'.

The OOPE incurred by family on delivery care, percent families who had borrowed money/sold property for meeting delivery care expenses, percent JSY beneficiary families/women are the main outcome measures of this study. Expenditure incurred by the woman/family on transportation was obtained only for institutional deliveries. If there was no expenditure on transportation it was coded as ' 0 ', else the actual expenditure was coded, up to a maximum of Rs. $89,999^{\text {a }}$. Delivery care expenditures (irrespective of place and type) include: antenatal care (ANC), delivery, and medicines during the period [23]. If no expenditure was incurred for delivery, it was coded as Rs. '0', otherwise the actual expenditure was coded up to a maximum of Rs. $99,996^{\mathrm{b}}$. By adding expenditures on transportation and delivery care we have computed a new variable, 'out-of-pocket expenditure (OOPE) of a delivery'.

States and union territories of India were grouped according to the National Rural Health Mission (NRHM) classification [24], as JSY compensation policies mainly vary according to this classification [14-16]:

- 10 High Focus - Non North Eastern (NE) states;

- 7 High Focus - NE states;

- 11 Non High Focus - Large states; and

- 6 Non High Focus - Small States \& Union Territories (UT).

We also measured variations in OOPE on normal/c-section delivery according to the following socio-demographic characteristics:

- Caste (scheduled caste, scheduled tribe, other backward caste, others);

- Maternal education (no education, 1-5 years, 6-11 years, and 12 years or more);

- Quintiles of household wealth index (poorest, second, middle, fourth, richest);

- Location of residence (rural, urban);

- Pregnant women's interaction with health worker [registered the pregnancy and got advice (at least once) on institutional delivery] (yes, no); and

- Got full ANC (yes, no).

\section{Statistical analysis}

The OOPE to family of delivery care was analyzed by estimating mean \& standard deviation (SD) and median \& inter-quartile range (IQR) values, because OOPE on delivery care data were heavily skewed. Chi-square and one-way analysis of variance (ANOVA) tests were used to test significance of difference between proportions and means respectively. We applied weights for the state in entire analysis. Analysis was undertaken in SPSS-19.

\section{Results}

The response rate of women who had a live/still birth between January 2007 and December 2008 in DLHS-3 was 93\% $(\mathrm{N}=92,563)$. Out of these women, data on OOPE for delivery care were available for 83,510 (90.2\%), and information on OOPE and type of delivery and place of delivery was available for 83,493 (90.2\%). The mean OOPE to family, only on ANC and delivery care for all births in India in 2007/08 was Rs. 2,037 (SD=4,509) and median of Rs. 500 (IQR=150-2,000). Mean expenditure exclusively on transportation for the 36,524 (39\%) women who had an institutional delivery was Rs. 322 ( $\mathrm{SD}=893)$, median of Rs. 150 (IQR=50-400). Mean (total) OOPE to family for maternity/delivery care (transportation + ANC+ delivery expenditure) was Rs. 2,169 (SD=4,647) with a median of Rs. 600 (IQR=200-2,000).

\section{Flow chart}

Summary profile of delivery care in India by type (normal/caesarean-section) and location private institution/private institution/home), in 2007-08.

Figure 1 provides a summary profile of OOPEs associated with delivery care in India according to type and place of delivery. Of all the deliveries in India in 2007$08,90 \%$ were classified as 'normal', $8 \%$ as 'c-section' and $2 \%$ as 'instrument/assisted'. The breakdown of the $90 \%$ normal deliveries by location of delivery was as follows: home (52\%); government hospital (25\%); private hospital (12\%); and others/NGOs (1\%). The breakdown of the $8 \%$ of c-sections was as follows: private institution (5\%); public institution (3\%); and others (0.4\%). The mean OOPE associated with a c-section birth was eight times that for a normal delivery, and high expenditures associated with these c-sections forced almost one-intwo women/families to borrow money. Mean OOPE of a normal delivery in public institution (Rs. 1,624) was three times that for a home delivery (Rs. 466), while a normal delivery in a private institution (Rs. 4,458) was three times that occurring in a public institution. One in every four women/families who had a normal delivery at home borrowed money, even though mean expenditure was only Rs. 466. One in every three women who had a normal delivery in public/private institution borrowed money. The JSY programme reach was mainly confined to public institution deliveries (43\%) with almost negligible reach to private institution $(6 \%)$ or home $(3 \%)$ deliveries. 


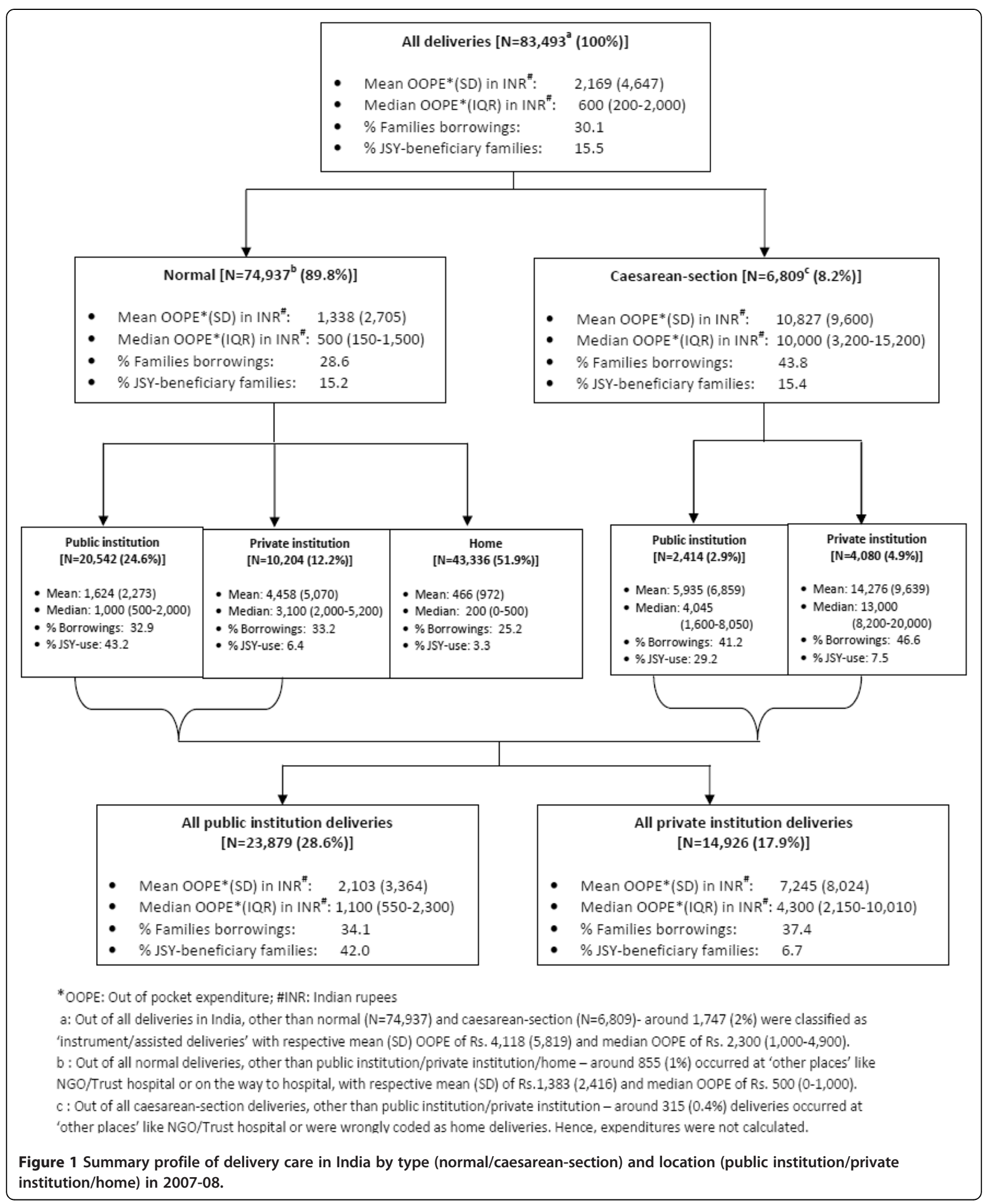


State level variations in OOPE, borrowings and JSY use for normal deliveries at public and private institutions and at home

Figure 2 presents mean OOPE, percent borrowings and percent JSY beneficiaries of a normal delivery in a public institution by state/UT (see Tables 1 and 2 for more detailed information). With large interstate variations, mean OOPE of a normal delivery in a public institution was least expensive (Rs. 381) in Daman \& Diu and most expensive in Manipur (Rs. 3,984), with a national average of Rs. 1,624 . In only nine out of 34 states/UTs, median OOPE was less than the JSY compensation amount of Rs. 700 (Table 1). Mean OOPE is not the sole determinant of families having to borrow money. For example, despite high mean OOPE (Rs. 3,230) in Arunachal Pradesh, only 8 percent families opted for borrowing, while despite a low mean OOPE (Rs. 1,769) in West Bengal, a large proportion (60\%) of families opted to borrowings. There were considerable state-wise variations in percent JSY beneficiaries even among the ten high focus- non NE states (76\% in Madhya Pradesh and $5 \%$ in Jammu \& Kashmir), when $100 \%$ these women are technically eligible to receive JSY benefit. Among the high focus - NE states, Assam did well in terms of JSY outreach followed by Mizoram. In non high focus states/ UTs, JSY use was generally low.

Figure 3 presents mean OOPE, percent borrowings and percent JSY beneficiaries for a normal delivery in a private institution by state/UT (see Tables 1 and 2 for additional data). Excluding Bihar, Lakshadweep, Delhi and Arunachal
Pradesh - mean OOPE for a normal delivery in the remaining states ranged from Rs. 3,000-8,000. Irrespective of mean OOPE, these deliveries were generally associated with higher borrowings and fewer JSY beneficiaries. At national level, only $6 \%$ of these deliveries received JSY benefit. Borrowings for these deliveries were high in Bihar, Orissa and Andhra Pradesh, while percent borrowings were lower in Maharashtra, Meghalaya and Mizoram. Irrespective of NRHM classification of states/ UTs, JSY reach to deliveries in private institutions was generally poor across all the states, excluding Tamil Nadu, Andhra Pradesh and Mizoram, where more than $20 \%$ received JSY benefit.

Figure 4 presents mean OOPE, percent borrowings and percent JSY beneficiaries for a normal delivery at home by state/UT (see Tables 1 and 2 for additional data). Mean OOPE for a normal delivery at home was Rs. 466, with one-fourth of women/families requiring to borrow money, while a negligible (3\%) proportion of them received the JSY benefit. Mean OOPE of a home delivery across the states/UTs may broadly be divided into three broad groups: less than Rs. 500 in 16 states; between Rs. 500-1,000 in 16; and more than Rs. 1000 in two states. High mean OOPE of these deliveries were generally associated with high borrowings and poor JSY outreach (less than $10 \%$ in 29 of the 34 states/UTs).

\section{State level variations in OOPE, borrowings and JSY use} for c-section deliveries at public and private institutions Data on mean OOPE, percent borrowings and percent JSY beneficiaries for $\mathrm{c}$-section deliveries at public and private

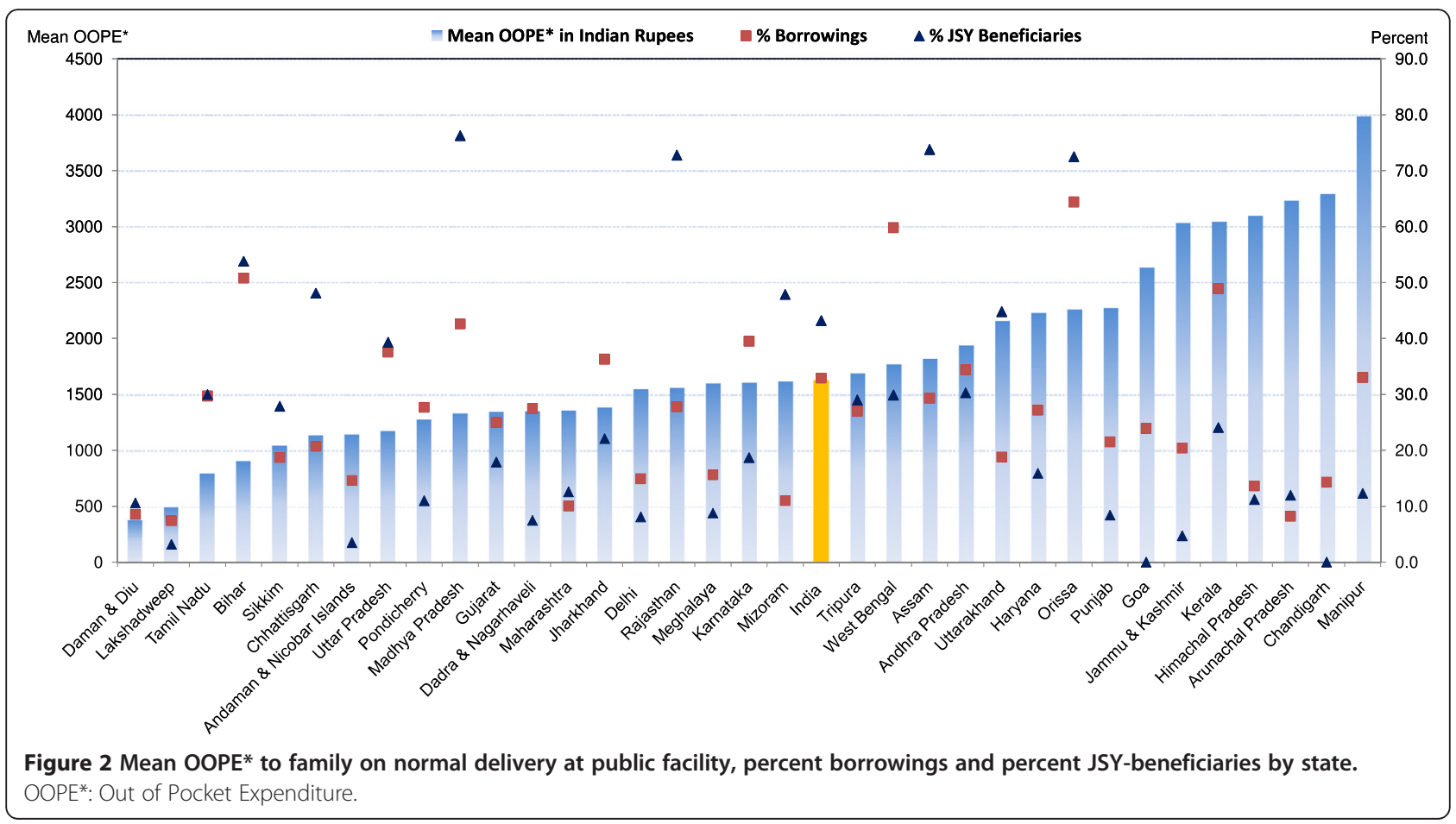


Table 1 Mean and median out-of-pocket-expenditure (OOPE) of normal vaginal deliveries by location and state/UT

\begin{tabular}{|c|c|c|c|c|c|c|c|c|c|}
\hline \multirow[t]{2}{*}{ STATE } & \multirow{2}{*}{$\begin{array}{l}\% \text { of all } \\
\text { deliveries }\end{array}$} & \multicolumn{4}{|c|}{ Mean OOPE in INR (SD) } & \multicolumn{4}{|c|}{ Median OOPE in INR (IQR) } \\
\hline & & Public & Private & Home & All normal & Public & Private & Home & All normal \\
\hline \multicolumn{10}{|c|}{ High Focus - Non NE } \\
\hline Jammu \& Kashmir & 82.4 & $3,028(2443)$ & $5,090(4468)$ & $760(1112)$ & $1,920(2441)$ & $2,500(1300-4000)$ & $3,950(2226-6164)$ & $400(0-1000)$ & $1,000(300-2800)$ \\
\hline Himachal Pradesh & 84.1 & $3,093(2785)$ & $6,768(6471)$ & $630(920)$ & $1,752(2823)$ & $2,400(1200-4000)$ & $5,316(3916-8347)$ & $500(0-800)$ & $600(200-200)$ \\
\hline Uttarakhand & 93.0 & $2,156(2060)$ & $5,472(3702)$ & $505(630)$ & $1,234(2038)$ & $1,500(1000-3000)$ & $5,071(3000-7000)$ & $500(100-600)$ & $500(200-1053)$ \\
\hline Rajasthan & 95.2 & $1,557(1413)$ & 3,522 (2981) & $504(1945)$ & $1,209(2038)$ & $1,200(750-1900)$ & $2,800(1742-4522)$ & $300(100-500)$ & $700(250-1500)$ \\
\hline Uttar Pradesh & 94.1 & $1,172(1707)$ & $3,623(5370)$ & $320(633)$ & $811(2261)$ & $750(500-1250)$ & $2,200(1200-4100)$ & $184(18-400)$ & $251(51-700)$ \\
\hline Bihar & 93.9 & $904(1188)$ & $2,961(3020)$ & $615(1103)$ & $888(1545)$ & $600(400-1033)$ & $2,062(1200-3500)$ & $400(200-600)$ & $500(200-1000)$ \\
\hline Jharkhand & 94.4 & $1,382(2036)$ & $3,742(3157)$ & $440(605)$ & $754(1462)$ & $800(463-1620)$ & $3,005(1900-5000)$ & $300(200-500)$ & $300(200-600)$ \\
\hline Orissa & 82.8 & $2,260(2408)$ & 4,261 (3375) & $316(801)$ & $1,229(2069)$ & $1,700(1050-2531)$ & $3,300(1587-5936)$ & $0(0-400)$ & $500(0-1650)$ \\
\hline Chhattisgarh & 94.6 & $1,135(1783)$ & $4,251(3652)$ & $179(323)$ & $480(1351)$ & $670(320-1100)$ & $3,500(1929-5712)$ & $0(0-300)$ & $60(0-500)$ \\
\hline Madhya Pradesh & 94.6 & 1,331 (1954) & 4,965 (5777) & 574 (962) & $1,217(2284)$ & $800(500-1500)$ & $3,254(2000-5500)$ & $300(20-700)$ & $600(200-1300)$ \\
\hline \multicolumn{10}{|l|}{ High Focus - NE } \\
\hline Sikkim & 83.7 & $1,043(710)$ & $7,700^{*}(2105)$ & $148(298)$ & $573(874)$ & $977(500-1500)$ & $7,700 *(6200-9200)$ & $0(0-100)$ & $300(0-900)$ \\
\hline Arunachal Pradesh & 94.7 & $3,230(4697)$ & 9,208 (11317) & $518(1319)$ & $1,844(3942)$ & $2,000(880-4014)$ & $7,152(2200-10197)$ & $0(0-500)$ & $500(90-2070)$ \\
\hline Manipur & 90.8 & 3,984 (3731) & 7,617 (4471) & 419 (712) & $1,870(3209)$ & $3,300(2200-5029)$ & $7,500(4397-10000)$ & $200(0-500)$ & $500(100-2500)$ \\
\hline Mizoram & 94.6 & $1,616(2276)$ & 6,435 (4180) & $350(492)$ & $1,289(2242)$ & $1,050(650-1700)$ & $5,300(3330-9521)$ & $200(0-500)$ & $700(200-1500)$ \\
\hline Tripura & 91.3 & $1,688(2074)$ & $3,824(3435)$ & $112(251)$ & $700(1530)$ & $1,000(600-2000)$ & $3,056(822-7841)$ & $0(0-150)$ & $150(0-800)$ \\
\hline Meghalaya & 89.7 & $1,600(2373)$ & $5,024(5778)$ & $225(596)$ & 664 (1947) & $900(400-2000)$ & 2,832 (1469-5967) & $0(0-300)$ & $150(0-500)$ \\
\hline Assam & 91.3 & $1,818(3362)$ & $6,280(5593)$ & $194(477)$ & 918 (2517) & $1,200(700-2100)$ & $5,000(2400-8211)$ & $0(0-250)$ & $220(0-1000)$ \\
\hline \multicolumn{10}{|c|}{ Non High Focus - Large } \\
\hline Punjab & 83.9 & 2,272 (1886) & 3,517 (2237) & $974(876)$ & 2,172 (2044) & $2,000(1030-3000)$ & $3,050(2100-4400)$ & $800(500-1100)$ & $1,650(800-3000)$ \\
\hline Haryana & 86.3 & 2,228 (3576) & $5,902(5996)$ & $844(1508)$ & 2,415 (4186) & $1,000(400-2800)$ & 4,839 (3000-7000) & $500(200-1000)$ & $900(250-3015)$ \\
\hline Delhi & 81.1 & $1,547(2898)$ & 10,372 (9916) & 945 (1570) & $3,491(6618)$ & $600(150-1632)$ & $8,000(5000-12184)$ & $600(300-1100)$ & $1,000(400-3623)$ \\
\hline Gujarat & 90.7 & $1,344(1969)$ & 3,267 (3956) & $298(529)$ & $1,511(2796)$ & $800(250-1852)$ & $2,200(1446-3877)$ & $150(0-380)$ & $560(101-2000)$ \\
\hline Maharashtra & 89.2 & 1,356 (1878) & 4,345 (5569) & $641(1090)$ & $2,043(3743)$ & $700(332-1700)$ & $3,052(2000-5050)$ & $300(0-1000)$ & $1,000(300-2500)$ \\
\hline Andhra Pradesh & 71.4 & $1,937(2280)$ & $5,071(5502)$ & $1,070(2014)$ & 2,589 (3917) & $1,100(600-2150)$ & $3,472(2100-5482)$ & $500(100-1500)$ & $1,500(500-3100)$ \\
\hline Karnataka & 83.9 & $1,604(1791)$ & 4,667 (4941) & 756 (1174) & $2,190(3383)$ & $1,100(600-2050)$ & $3,300(2050-5488)$ & $500(50-1000)$ & $1,100(500-3000)$ \\
\hline Goa & 71.3 & 2,630 (3193) & 7,685 (4848) & $319(453)$ & 4,859 (4797) & $2,000(300-3600)$ & $6,719(4365-10000)$ & $67(0-780)$ & 4,037 (1182-7026) \\
\hline Kerala & 65.9 & $3,040(2676)$ & 6,624 (5282) & $1,394(2106)$ & $5,332(4843)$ & $2,100(1100-4210)$ & $5,160(4000-8000)$ & $150(75-3478)$ & $4,800(2500-6377)$ \\
\hline Tamil Nadu & 73.7 & $792(1142)$ & 4,926 (4808) & $102(365)$ & $2,062(3442)$ & $418(50-1030)$ & 3,910 (2549-5831) & $0(0-0)$ & $850(100-3000)$ \\
\hline West Bengal & 85.6 & $1,769(2163)$ & $5,082(4970)$ & $568(863)$ & 1,197 (2050) & $1,096(568-2100)$ & 4,042 (2010-6172) & $300(100-600)$ & $500(200-1300)$ \\
\hline
\end{tabular}


Table 1 Mean and median out-of-pocket-expenditure (OOPE) of normal vaginal deliveries by location and state/UT (Continued)

\begin{tabular}{|c|c|c|c|c|c|c|c|c|c|}
\hline \\
\hline \multicolumn{10}{|c|}{$\begin{array}{l}\text { Non High Focus - Small states \& UTs } \\
\text { Chandigarh } \\
87.4\end{array}$} \\
\hline Daman \& Diu & 83.3 & $381(780)$ & $4,543(2804)$ & $490(666)$ & $1,908(2620)$ & $100(1-287)$ & $4,100(2500-5316)$ & $207(100-500)$ & $500(100-3000)$ \\
\hline Dadra \& Nagarhaveli & 95.3 & $1,351(1662)$ & $6,063(2960)$ & $616(1013)$ & $1,536(2440)$ & $545(50-2127)$ & $7,028(4191-7447)$ & $200(100-700)$ & $300(100-2000)$ \\
\hline Lakshadweep & 76.7 & $494(1645)$ & $21,750(14947)$ & $233(331)$ & $1,570(5855)$ & $50(20-60)$ & $17,000(10375-37875)$ & $0(0-500)$ & $50(0-500)$ \\
\hline Pondicherry & 66.4 & $1,276(1707)$ & $6,500(3478)$ & $75^{*}(103)$ & $2,565(3207)$ & $563(150-1700)$ & $5,255(4100-8670)$ & $75^{*}(0-75)$ & $1091(311-3858)$ \\
\hline Andaman \& Nicobar Islands & 86.9 & $1,143(3028)$ & $6,463(5617)$ & 909 (1965) & 1,191 (2929) & $100(0-1000)$ & $9,500(0-9992)$ & $0(0-899)$ & $99(0-1000)$ \\
\hline India - Total & 89.7 & 1,624 & $4,458(5070)$ & $466(972)$ & $1,338(2705)$ & $1,000(500-2000)$ & $3,100(2000-5200)$ & $200(0-500)$ & $500(150-1500)$ \\
\hline
\end{tabular}


Table 2 Percent borrowings and percent JSY beneficiaries by delivery type, location and state/UT

\begin{tabular}{|c|c|c|c|c|c|c|c|c|c|c|c|c|c|c|}
\hline \multirow{3}{*}{ STATE } & \multicolumn{7}{|c|}{$\%$ Borrowings } & \multicolumn{7}{|c|}{$\%$ JSY beneficiaries } \\
\hline & \multicolumn{4}{|c|}{ Normal } & \multicolumn{3}{|c|}{ Caesarian } & \multicolumn{4}{|c|}{ Normal } & \multicolumn{3}{|c|}{ Caesarian } \\
\hline & Public & Private & Home & $\begin{array}{l}\text { All } \\
\text { normal }\end{array}$ & Public & Private & $\begin{array}{l}\text { All } \\
\text { c-section }\end{array}$ & Public & Private & Home & $\begin{array}{l}\text { All } \\
\text { normal }\end{array}$ & Public & Private & $\begin{array}{l}\text { All } \\
\text { c-section }\end{array}$ \\
\hline \multicolumn{15}{|l|}{ High Focus - Non NE } \\
\hline Jammu \& Kashmir & 20.4 & 34.2 & 12.8 & 17.4 & 25.9 & 26.8 & 25.7 & 4.7 & 1.8 & 0.8 & 2.6 & 4.9 & 3.6 & 4.5 \\
\hline Himachal Pradesh & 13.6 & 23.6 & 9.9 & 12.0 & 25.9 & 41.3 & 32.0 & 11.2 & 3.6 & 2.3 & 5.4 & 9.9 & 1.6 & 6.2 \\
\hline Uttarakhand & 18.8 & 26.9 & 9.9 & 13.2 & 34.1 & 50.8 & 43.3 & 44.8 & 1.4 & 1.9 & 10.9 & 31.7 & 1.7 & 13.5 \\
\hline Rajasthan & 27.8 & 29.8 & 15.4 & 22.1 & 33.7 & 42.4 & 36.6 & 72.8 & 4.4 & 1.5 & 32.3 & 55.1 & 5.4 & 28.3 \\
\hline Uttar Pradesh & 37.6 & 44.2 & 27.4 & 30.8 & 45.3 & 56.1 & 51.8 & 39.3 & 2.4 & 1.2 & 6.2 & 30.0 & 3.0 & 8.0 \\
\hline Bihar & 50.8 & 52.8 & 47.9 & 48.9 & 61.3 & 68.6 & 65.1 & 53.8 & 2.7 & 1.3 & 13.5 & 33.9 & 4.1 & 9.3 \\
\hline Jharkhand & 36.3 & 32.8 & 30.3 & 30.9 & 53.6 & 54.0 & 53.4 & 22.1 & 2.3 & 1.6 & 3.8 & 17.9 & 5.1 & 6.9 \\
\hline Orissa & 64.4 & 49.5 & 29.6 & 44.5 & 71.9 & 59.2 & 61.3 & 72.5 & 17.5 & 9.5 & 35.5 & 66.1 & 9.9 & 43.1 \\
\hline Chhattisgarh & 20.7 & 31.8 & 10.0 & 12.4 & 52.6 & 46.4 & 44.8 & 48.1 & 6.8 & 4.0 & 10.5 & 34.2 & 3.6 & 14.3 \\
\hline Madhya Pradesh & 42.6 & 31.7 & 31.8 & 37.6 & 59.7 & 44.4 & 51.4 & 76.3 & 9.8 & 4.1 & 42.9 & 70.6 & 3.7 & 34.4 \\
\hline \multicolumn{15}{|l|}{ High Focus - NE } \\
\hline Sikkim & 18.7 & $25.0^{*}$ & 11.4 & 14.8 & 21.4 & 15.4 & 20.0 & 27.9 & $0.0^{*}$ & 19.4 & 23.2 & 26.2 & 7.7 & 21.8 \\
\hline Arunachal Pradesh & 8.2 & 0.0 & 1.6 & 4.6 & 9.6 & 12.5 & 9.7 & 12.0 & 5.3 & 1.4 & 6.1 & 5.8 & 25.0 & 8.1 \\
\hline Manipur & 33.0 & 36.2 & 8.2 & 17.1 & 40.0 & 40.8 & 39.6 & 12.3 & 2.6 & 3.3 & 5.7 & 13.8 & 6.6 & 9.7 \\
\hline Mizoram & 11.0 & 13.2 & 3.9 & 7.9 & 27.1 & 9.1 & 22.2 & 47.9 & 47.4 & 5.0 & 29.0 & 41.7 & 9.1 & 34.9 \\
\hline Tripura & 27.0 & 42.9 & 7.3 & 15.4 & 39.4 & 18.2 & 33.3 & 29.0 & 0.0 & 2.0 & 12.1 & 18.2 & 0.0 & 13.3 \\
\hline Meghalaya & 15.6 & 10.0 & 8.9 & 10.4 & 23.5 & 18.2 & 15.4 & 8.8 & 2.0 & 1.3 & 2.7 & 5.9 & 8.3 & 3.8 \\
\hline Assam & 29.3 & 26.0 & 11.9 & 18.1 & 41.0 & 28.8 & 34.4 & 73.8 & 14.2 & 3.0 & 26.9 & 59.6 & 3.9 & 33.9 \\
\hline \multicolumn{15}{|c|}{ Non High Focus - Large } \\
\hline Punjab & 21.5 & 25.4 & 36.2 & 29.4 & 38.1 & 44.9 & 43.6 & 8.4 & 1.0 & 1.4 & 2.5 & 10.6 & 1.1 & 3.4 \\
\hline Haryana & 27.2 & 34.1 & 30.5 & 31.0 & 34.2 & 49.8 & 46.3 & 15.9 & 3.0 & 3.7 & 5.4 & 11.8 & 2.0 & 4.2 \\
\hline Delhi & 14.9 & 19.3 & 27.7 & 20.8 & 18.8 & 25.9 & 23.2 & 8.1 & 1.4 & 0.3 & 3.5 & 4.7 & 1.2 & 2.6 \\
\hline Gujarat & 25.0 & 30.8 & 18.4 & 24.2 & 31.6 & 38.2 & 36.8 & 17.9 & 10.6 & 6.2 & 10.4 & 17.5 & 5.3 & 7.9 \\
\hline Maharashtra & 10.0 & 13.4 & 6.8 & 9.9 & 15.3 & 18.4 & 17.0 & 12.6 & 2.9 & 11.3 & 9.0 & 15.3 & 3.0 & 6.6 \\
\hline Andhra Pradesh & 34.4 & 47.7 & 30.9 & 37.3 & 51.6 & 60.3 & 58.2 & 30.3 & 20.3 & 6.0 & 18.6 & 41.4 & 18.1 & 23.1 \\
\hline Karnataka & 39.5 & 34.6 & 26.3 & 33.3 & 50.9 & 42.2 & 45.7 & 18.7 & 10.1 & 9.7 & 13.1 & 19.6 & 14.3 & 16.6 \\
\hline Goa & 23.9 & 22.5 & 33.3 & 23.9 & 50.0 & 7.4 & 18.9 & 0.0 & 0.0 & 0.0 & 0.0 & 0.0 & 3.7 & 2.7 \\
\hline Kerala & 48.9 & 31.7 & 25.0 & 38.1 & 62.7 & 45.8 & 51.2 & 24.1 & 7.2 & 12.5 & 13.5 & 26.1 & 4.8 & 11.6 \\
\hline Tamil Nadu & 29.7 & 41.4 & 8.0 & 32.0 & 44.2 & 59.4 & 53.9 & 30.0 & 22.7 & 23.9 & 27.3 & 43.2 & 23.7 & 30.2 \\
\hline West Bengal & 59.8 & 41.2 & 47.1 & 51.5 & 60.8 & 44.3 & 52.5 & 29.9 & 10.4 & 15.1 & 20.1 & 24.6 & 9.2 & 16.3 \\
\hline \multicolumn{15}{|c|}{ Non High Focus - Small States \& UTs } \\
\hline Chandigarh & 14.3 & 16.7 & 30.4 & 19.7 & 60.0 & $0.0^{*}$ & 37.5 & 0.0 & 0.0 & 0.0 & 0.0 & 0.0 & $0.0^{*}$ & 0.0 \\
\hline Daman \& Diu & 8.5 & 23.9 & 11.0 & 15.3 & 25.0 & 44.7 & 41.3 & 10.6 & 2.3 & 0.0 & 2.8 & 0.0 & 2.6 & 2.2 \\
\hline Dadra \& Nagarhaveli & 27.5 & 26.1 & 35.1 & 32.3 & $0.0^{*}$ & 20.0 & 12.5 & 7.5 & 8.7 & 0.0 & 3.1 & 0.0 & 0.0 & 0.0 \\
\hline Lakshadweep & 7.4 & 0.0 & 0.0 & 5.7 & 0.0 & 23.8 & 13.5 & 3.2 & 0.0 & 0.0 & 2.5 & 6.3 & 0.0 & 2.7 \\
\hline Pondicherry & 27.7 & 20.0 & $0.0^{*}$ & 25.6 & 30.0 & 18.0 & 24.5 & 11.0 & 1.7 & 0.0 & 8.2 & 16.7 & 0.0 & 9.1 \\
\hline $\begin{array}{l}\text { Andaman \& Nicobar } \\
\text { Islands }\end{array}$ & 14.6 & 0.0 & 10.9 & 13.1 & 16.7 & 66.7 & 20.7 & 3.5 & 0.0 & 1.8 & 2.9 & 4.2 & 0.0 & 3.4 \\
\hline India - Total & 32.9 & 33.2 & 25.2 & 28.6 & 41.2 & 46.6 & 43.8 & 43.2 & 6.4 & 3.3 & 15.2 & 29.2 & 7.5 & 15.4 \\
\hline
\end{tabular}




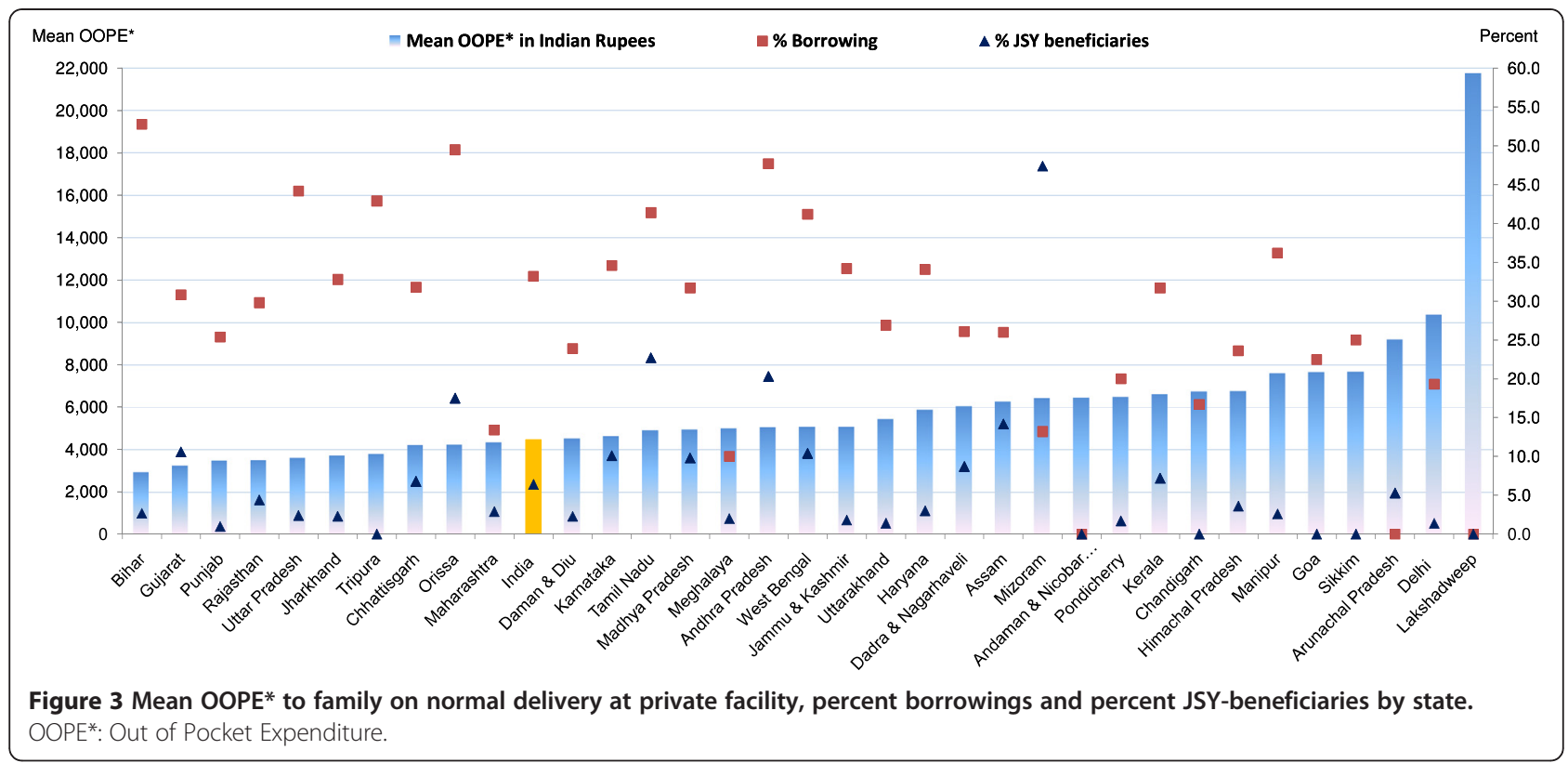

institutions by state/UT is presented in Figures 5 and 6 and Tables 2 and 3. Mean OOPE for a c-section in a public institution was Rs. 5,935, ranging from Rs. 678 in Daman \& Diu to Rs. 13,165 in Uttarakhand. These deliveries, despite occurring in public institutions, have forced more than two-fifths (41\%) of families to borrow money, with less than one-third (29\%) receiving the JSY-benefit. Percent families borrowings for these public institution c-section deliveries were $60 \%$ or more in the states of: Chandigarh, Bihar, Orissa, West Bengal and Kerala; while percentage of families benefitting through JSY program was less than 10\% in Daman \& Diu, Andaman \& Nicobar Islands, Goa, Chandigarh, Arunachal Pradesh, Jammu \& Kashmir, Delhi, Himachal Pradesh, Meghalaya, Dadra \& Nagarhaveli and Lakshadweep. Mean OOPE for a c-section in a private institution was Rs. 14,276, ranging from Rs. 10,554 in Dadra \& Nagarhaveli to Rs. 39,424 in Andaman \& Nicobar Islands. For these c-sections almost half (47\%) of the families had to opt for borrowings with state/UT wise variation ranging from $7 \%$ to $69 \%$ (Tables 2 and 3). Use of the JSY

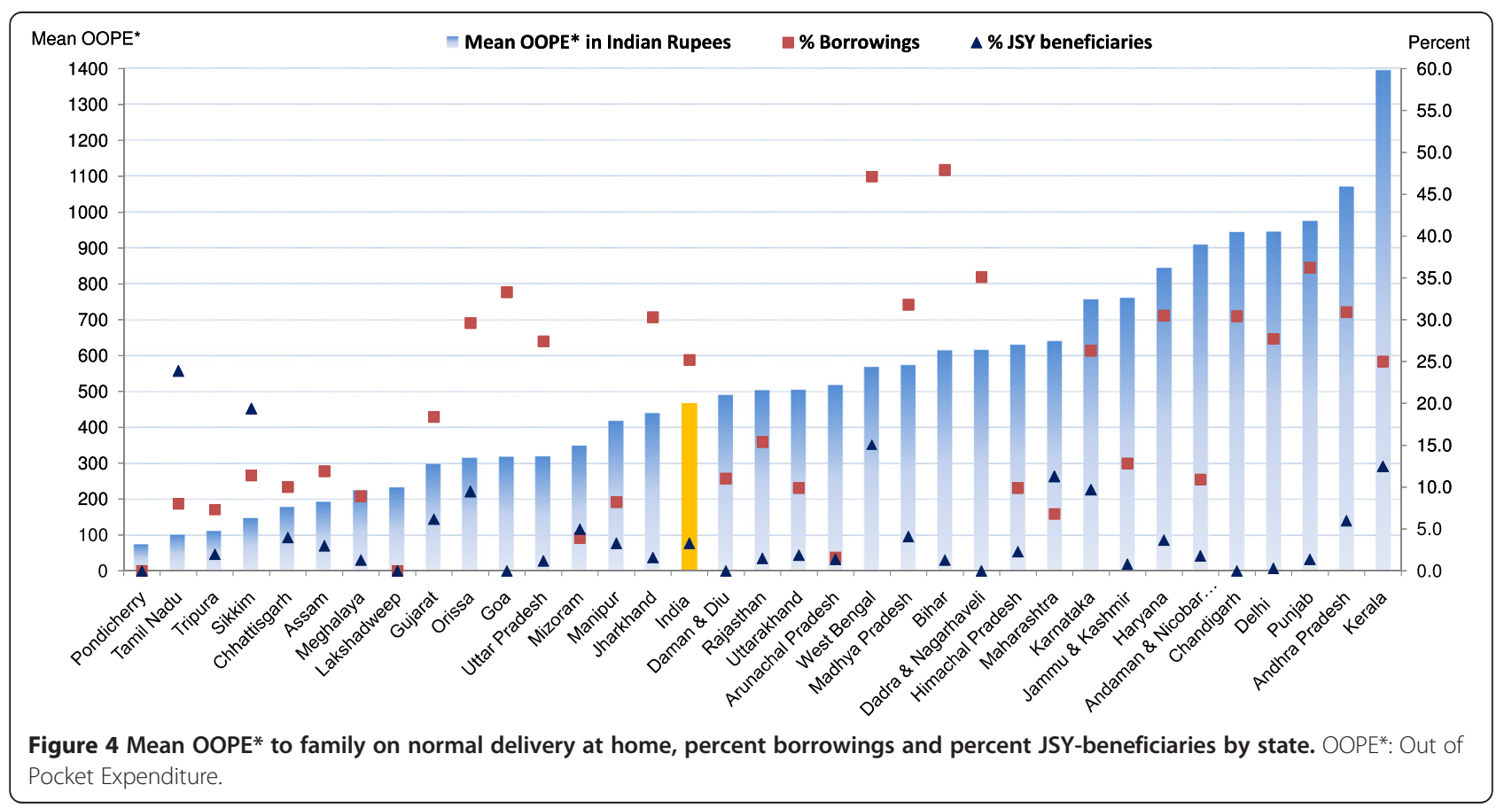




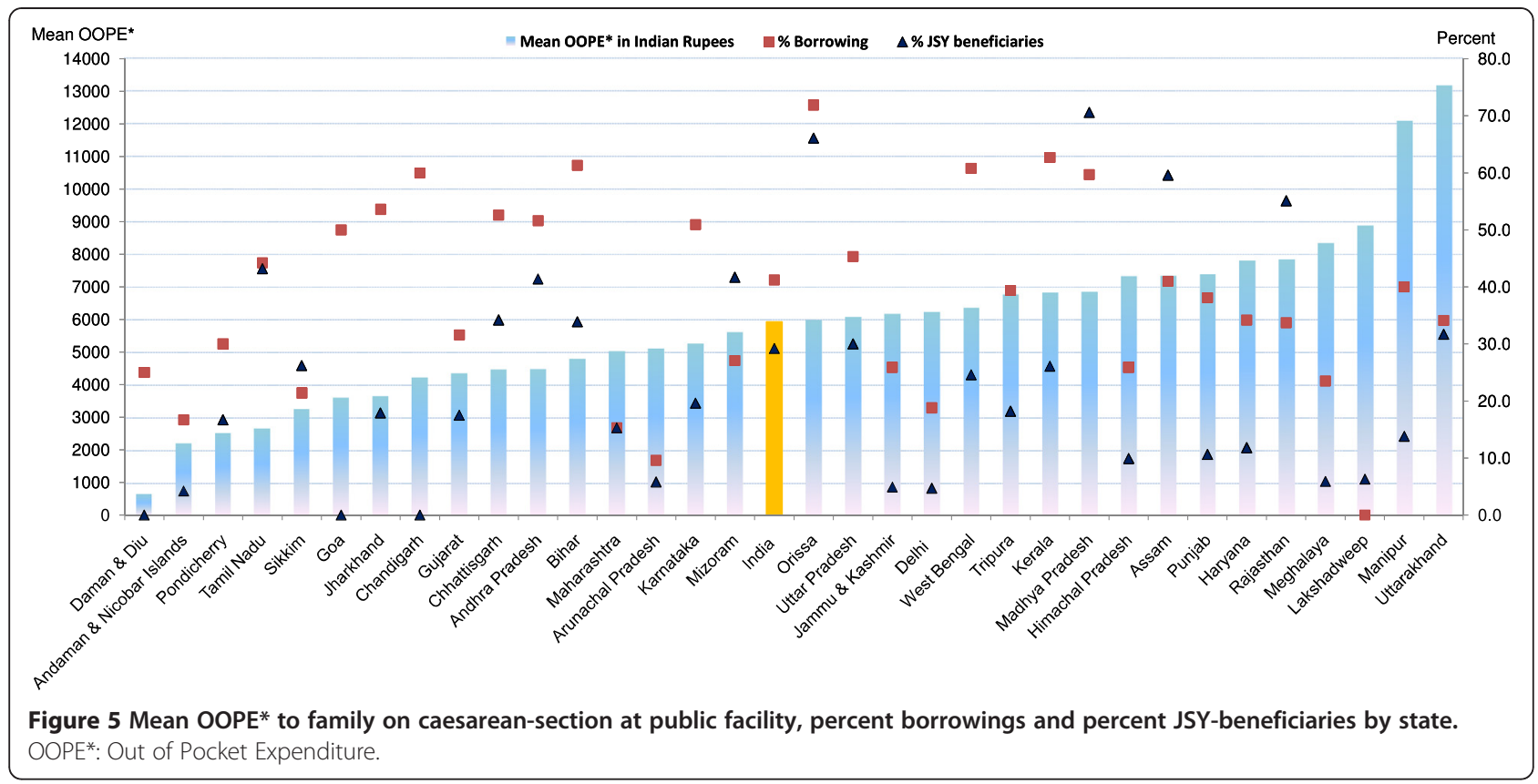

programme among women having a c-section in a private institution was $8 \%$ nationally, ranging from $0 \%$ to $25 \%$. Irrespective of NRHM classification of states/UTs, these deliveries were generally associated with higher borrowings and fewer JSY benefits.

\section{Socio-demographic variations in OOPE, borrowings and} JSY use for all normal/c-section deliveries in India

Variations in mean OOPE, percent borrowings and percent JSY beneficiaries - according to socio-demographic profiling of all normal and c-section deliveries in India are presented in Figures 7 and 8 (see Tables 4 and 5 for additional data). Mean OOPE of a normal delivery was significantly higher for other caste: Rs. 1,$996 ; \geq 12$ years educated: Rs. 3,429; and richest: Rs. 3,170 women, as compared to their scheduled tribe Rs. 805; illiterate Rs. 775; and poorest Rs. 605 counterparts. Higher literacy and wealth was associated with fewer borrowings but had no influence on use of the JSY-benefit. Mean OOPE of a normal delivery in urban areas (Rs. 2,290) was around two times the rural area (Rs. 1,163). Proportion borrowing and JSY reach did not differ significantly by rural/urban differentials. Mean OOPE

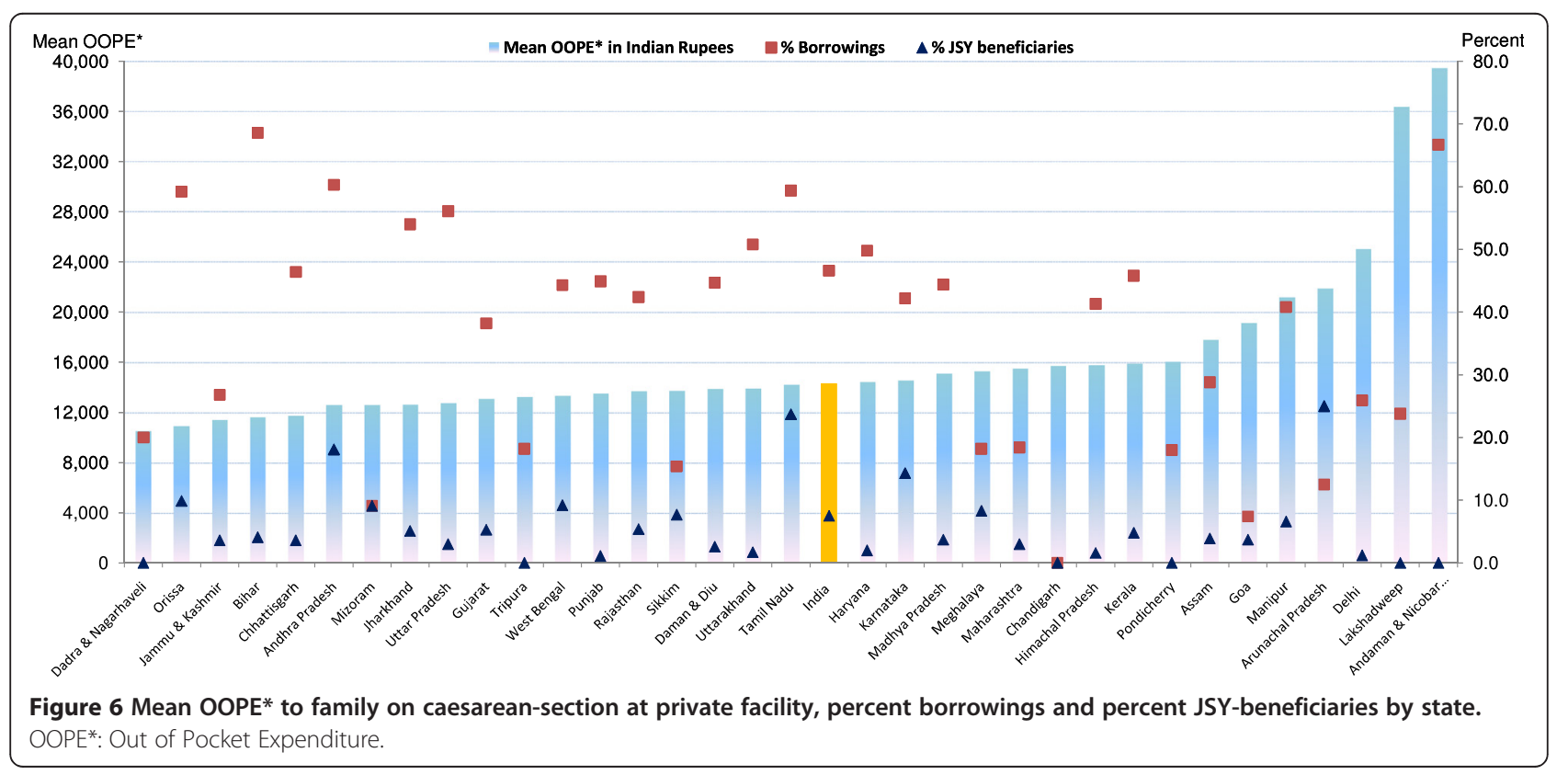


Table 3 Mean and median out-of-pocket-expenditure (OOPE) of caesarean-section deliveries by location and state/UT

\begin{tabular}{|c|c|c|c|c|c|c|c|}
\hline \multirow[t]{2}{*}{ STATE } & \multirow{2}{*}{$\begin{array}{l}\% \text { all } \\
\text { deliveries }\end{array}$} & \multicolumn{3}{|c|}{ Mean cost in INR (SD) } & \multicolumn{3}{|c|}{ Median cost in INR (IQR) } \\
\hline & & Public & Private & All c-section & Public & Private & All c-section \\
\hline \multicolumn{8}{|c|}{ High Focus - Non NE } \\
\hline Jammu \& Kashmir & 14.9 & $6,182(4879)$ & $11,428(6687)$ & $6,876(5621)$ & $5,010(2446-8930)$ & $11,000(5794-16000)$ & $5,300(2497-10078)$ \\
\hline Himachal Pradesh & 11.4 & $7,330(6677)$ & $15,791(10174)$ & $10,935(9436)$ & $5,500(3011-10084)$ & $15,326(7709-20407)$ & $8,000(3500-15790)$ \\
\hline Uttarakhand & 5.1 & $13,165(18021)$ & $13,916(10037)$ & $13,811(13406)$ & 8115 (3000-17308) & $15,500(5000-20395)$ & 12,109 (3867-20143) \\
\hline Rajasthan & 3.7 & $7,843(10375)$ & $13,718(9264)$ & $10,336(10224)$ & $5021(2000-9060)$ & $11,683(6495-20000)$ & $8,144(2634-15300)$ \\
\hline Uttar Pradesh & 4.6 & $6,097(6478)$ & $12,767(10377)$ & $10,614(10039)$ & 3,298 (1500-9828) & 10,500 (4089-17392) & $9,154(2500-15500)$ \\
\hline Bihar & 3.7 & $4,810(5446)$ & $11,644(8156)$ & $9,351(8257)$ & 2,881 (813-7189) & $10,256(5000-15235)$ & $8,100(2097-15000)$ \\
\hline Jharkhand & 3.9 & $3,670(3250)$ & $12,670(8481)$ & $11,069(8552)$ & $2,600(1344-5311)$ & 11,837 (7027-17000) & $10,034(3956-16000)$ \\
\hline Orissa & 10.1 & $5,990(6010)$ & $10,949(7412)$ & $6,286(6753)$ & $3,300(1700-10011)$ & 10,020 (4164-15414) & $3,273(1277-10200)$ \\
\hline Chhattisgarh & 4.6 & 4,477 (3533) & $11,790(6121)$ & $7,870(6520)$ & $2,745(1533-7070)$ & 10,702 (8004-15194) & $6,869(2092-11649)$ \\
\hline Madhya Pradesh & 3.7 & $6,855(7316)$ & $15,136(9221)$ & $11,152(9368)$ & 4,468 (1300-10074) & $15,014(10020-20060)$ & $10,050(2800-16027)$ \\
\hline \multicolumn{8}{|l|}{ High Focus - NE } \\
\hline Sikkim & 10.2 & $3,261(1957)$ & $13,748(10035)$ & $5,989(6997)$ & $2,657(2026-4028)$ & 10,752 (7006-17928) & $3,172(2108-6827)$ \\
\hline Arunachal Pradesh & 5.0 & $5,123(6478)$ & $21,905(47483)$ & 8,237 (20947) & $3,100(1015-8000)$ & $5,457(2915-8834)$ & $3,381(1276-8000)$ \\
\hline Manipur & 8.2 & $12,086(6710)$ & $21,158(8074)$ & $16,685(8883)$ & 10,702 (8083-15203) & $20,588(16820-25825)$ & $15,560(10187-20800)$ \\
\hline Mizoram & 4.8 & $5,615(3347)$ & $12,620(8148)$ & $7,093(5359)$ & $5,000(3433-7217)$ & $12,674(6321-15100)$ & 5,322 (4000-10080) \\
\hline Tripura & 6.3 & $6,774(4252)$ & $13,265(3156)$ & $8,036(4957)$ & 7,074 (3822-10009) & 12,201 (11104-14835) & 7,927 (4145-12005) \\
\hline Meghalaya & 3.2 & $8,346(14016)$ & $15,303(8279)$ & 7,489 (10636) & 3,478 (1362-10753) & 14,478 (8546-22772) & $2,456(472-11000)$ \\
\hline Assam & 5.9 & $7,342(7596)$ & $17,825(11868)$ & $10,445(10755)$ & $5,652(2300-10119)$ & 18,106 (7500-25000) & $7,000(2215-16272)$ \\
\hline \multicolumn{8}{|c|}{ Non High Focus - Large } \\
\hline Punjab & 13.6 & $7,383(5067)$ & $13,525(7028)$ & $12,029(7117)$ & 6,050 (3500-10168) & $12,962(10000-17144)$ & $11,008(6050-15487)$ \\
\hline Haryana & 10.5 & $7,807(7597)$ & $14,450(10765)$ & $13,017(10515)$ & 6,429 (1880-10438) & $15,000(5282-20100)$ & $12,374(4514-20000)$ \\
\hline Delhi & 14.2 & $6,246(11543)$ & $25,028(16415)$ & 17,525 (17233) & $3,050(1044-5932)$ & $21,395(13015-35000)$ & $13,016(3050-25762)$ \\
\hline Gujarat & 7.4 & 4,367 (7818) & 13,099 (9232) & $11,220(9539)$ & $2,330(200-5235)$ & $12,020(8120-15906)$ & $10,200(3300-15066)$ \\
\hline Maharashtra & 10 & $5,042(5012)$ & 15,509 (8498) & $12,175(8991)$ & $4,027(1500-6577)$ & $15,052(10050-20020)$ & $11,000(5018-17146)$ \\
\hline Andhra Pradesh & 27.6 & 4,499 (4400) & $12,602(6888)$ & $10,700(7269)$ & $3,158(1500-5100)$ & 10,300 (9099-15200) & $10,050(5100-15050)$ \\
\hline Karnataka & 14.0 & $5,271(5743)$ & $14,572(9519)$ & $10,903(9400)$ & $4,008(2000-6470)$ & $13,279(10005-18100)$ & 10,025 (3524-15100) \\
\hline Goa & 28.7 & 3,617 (3350) & $19,158(16120)$ & $15,034(15494)$ & 4,000 (416-5263) & $15,115(14175-21862)$ & $15,000(4818-17745)$ \\
\hline Kerala & 34.0 & $6,830(7551)$ & $15,933(10055)$ & 13,294 (10234) & $5,100(3253-7152)$ & $14,029(10274-19000)$ & 12,005 (6138-16000) \\
\hline Tamil Nadu & 24.9 & $2,674(4619)$ & $14,239(6819)$ & $10,295(8207)$ & $1,249(266-3082)$ & $14,963(10025-17143)$ & $10,050(2380-15100)$ \\
\hline West Bengal & 11.4 & $6,367(5118)$ & 13,357 (7800) & $10,131(7516)$ & $5,400(3066-8072)$ & $11,000(8150-16035)$ & $8,400(5054-12733)$ \\
\hline
\end{tabular}


Table 3 Mean and median out-of-pocket-expenditure (OOPE) of caesarean-section deliveries by location and state/UT (Continued)

\begin{tabular}{|c|c|c|c|c|c|c|c|}
\hline \multicolumn{8}{|c|}{ Non High Focus - Small states \& UTs } \\
\hline Chandigarh & 9.2 & $4,233(2246)$ & $15,750^{*}(12078)$ & $9,169(9436)$ & $3,790(2363-6545)$ & $20,100^{*}(2100-20100)$ & $5,030(2300-20100)$ \\
\hline Daman \& Diu & 15.4 & 678 (1094) & $13,893(6143)$ & $11,463(7594)$ & $77(0-1118)$ & $15,000(8546-20000)$ & $12,055(5011-16611)$ \\
\hline $\begin{array}{l}\text { Dadra \& } \\
\text { Nagarhaveli }\end{array}$ & 4.7 & - & $10,554(6612)$ & $10,554(6612)$ & - & $10,204(5244-16257)$ & $10,204(5244-16257)$ \\
\hline Lakshadweep & 23.3 & $8,876(19767)$ & $36,345(17837)$ & $25,323(22824)$ & $1,014(30-7668)$ & $29,604(24070-56986)$ & $24,521(1500-49561)$ \\
\hline Pondicherry & 33.3 & $2,542(3102)$ & $16,073(9096)$ & $8,433(9295)$ & $1,100(550-3000)$ & $15,000(10151-20056)$ & $5,890(1013-13318)$ \\
\hline $\begin{array}{l}\text { Andaman \& } \\
\text { Nicobar Islands }\end{array}$ & 12.2 & $2,228(3059)$ & $39,424(31749)$ & $5,514(13279)$ & $565(50-5000)$ & $39,775(17500-39775)$ & 797 (39-5042) \\
\hline India - Total & 8.2 & $5,935(6859)$ & 14,276 (9639) & $10,827(9600)$ & $4,045(1600-8050)$ & $13,000(8200-20000)$ & $10,000(3200-15200)$ \\
\hline
\end{tabular}




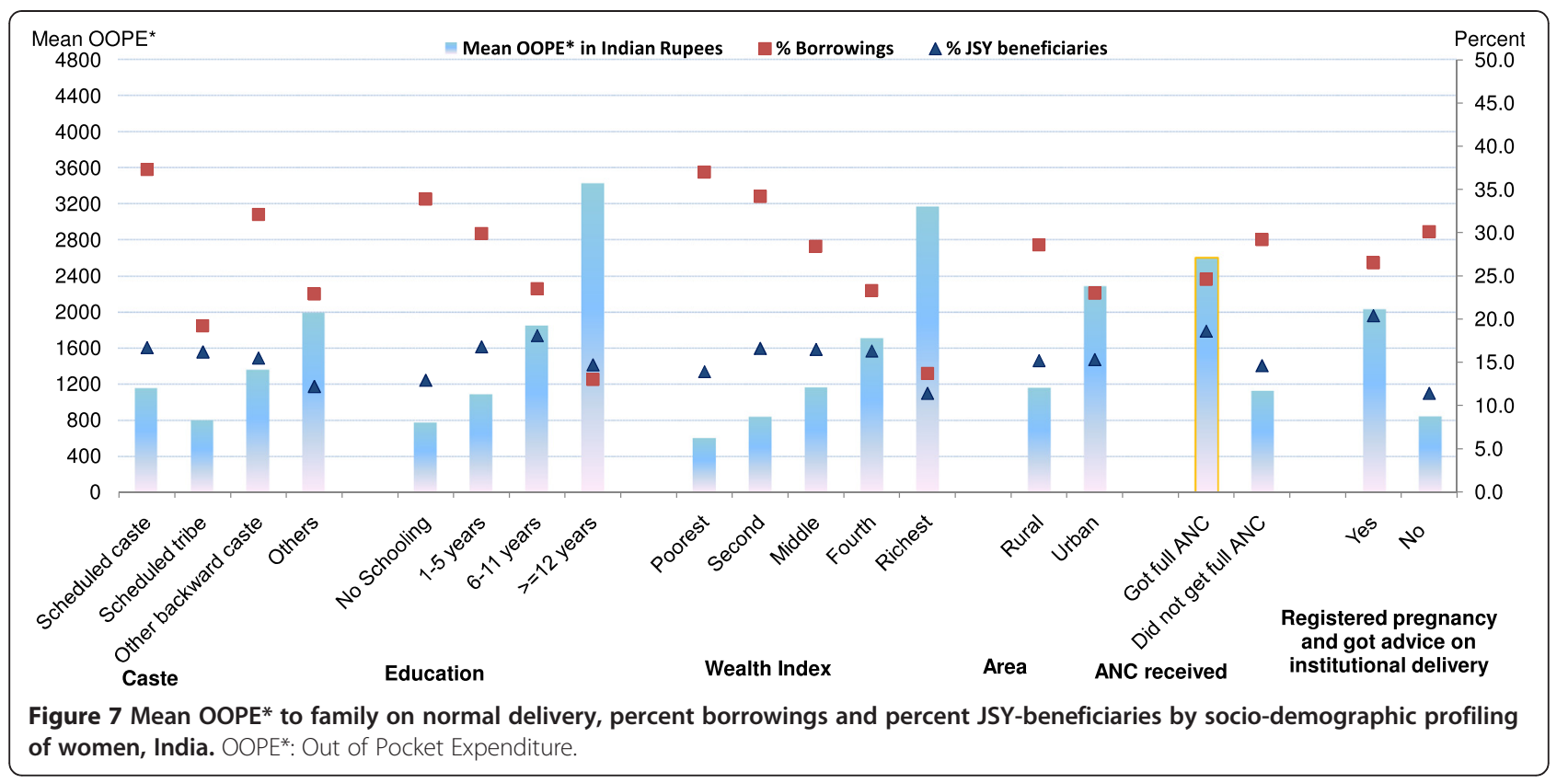

of a normal delivery was more than double in those who had full ANC or who interacted with a health worker during pregnancy as compared to their respective group counterparts. JSY reach and proportion borrowing did not differ significantly according to ANC use and women's interaction with health worker (Table 4). Excluding education and wealth index, in the remaining socio-demographic groups, variations in mean OOPE and \% borrowing were less evident among the c-section deliveries (Figure 8), as compared to normal deliveries, in India. The OOPE on csections did not differ significantly according to type of area (rural/urban), receiving full ANC care (yes/no) and pregnant woman's interaction with health worker (yes/no)
(Table 5). For poor and illiterate women, expenditures on c-sections were beyond their capacity to pay resulted in significantly more borrowings.

\section{Discussion}

In 2007-08, four years after the implementation of the JSY programme, half of all deliveries in India occurred at home. OOPE among women having institutional deliveries remained high, with considerable variation between the states/UTs. High OOPE due to institutional delivery forced one-third to half of the families to opt for borrowings, despite implementation of JSY programme to address this, reflecting both low use and the modest value for cash

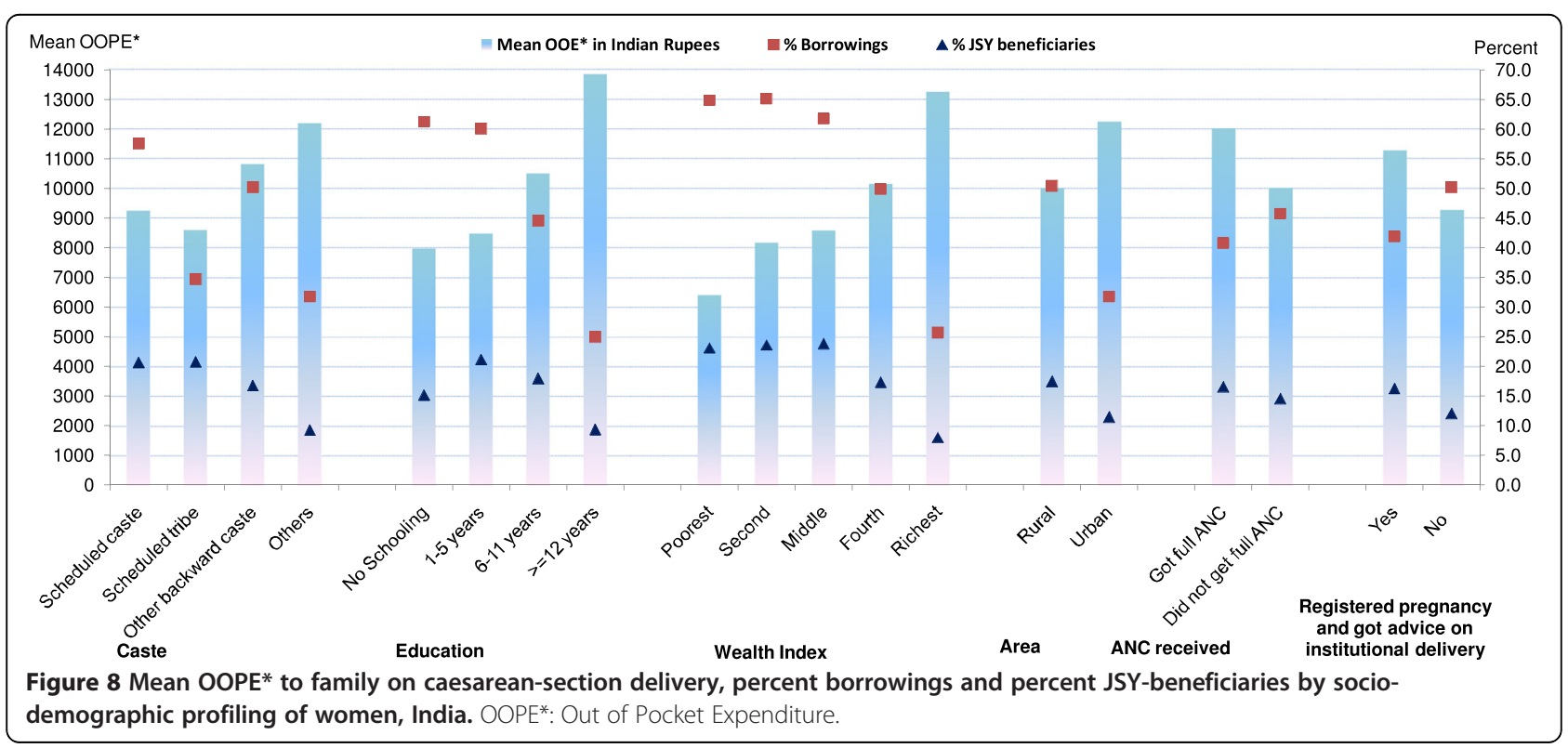


Table 4 Mean \& median out-of-pocket-expenditure (OOPE) of normal deliveries, percent borrowings and percent JSY-beneficiaries according to socio economic profile of women

\begin{tabular}{|c|c|c|c|c|c|c|c|c|c|c|c|c|c|}
\hline \multirow{2}{*}{$\begin{array}{l}\text { Socio-demographic } \\
\text { characteristics }\end{array}$} & \multicolumn{4}{|c|}{ Public institution } & \multicolumn{4}{|c|}{ Private institution } & \multicolumn{4}{|c|}{ Home } & \multirow{2}{*}{$\begin{array}{l}\text { Total } \\
\text { Women }\end{array}$} \\
\hline & $\begin{array}{l}\text { Mean } \\
\text { OOPE in } \\
\text { INR (SD) }\end{array}$ & $\begin{array}{l}\text { Median } \\
\text { OOPE in } \\
\text { INR (IQR) }\end{array}$ & \% Borrowing & $\begin{array}{l}\% \text { JSY } \\
\text { beneficiaries }\end{array}$ & $\begin{array}{l}\text { Mean } \\
\text { OOPE in } \\
\text { INR (SD) }\end{array}$ & $\begin{array}{l}\text { Median } \\
\text { OOPE in } \\
\text { INR (IQR) }\end{array}$ & $\%$ Borrowing & $\begin{array}{l}\% \text { JSY } \\
\text { beneficiaries }\end{array}$ & $\begin{array}{l}\text { Mean } \\
\text { OOPE in } \\
\text { INR(SD) }\end{array}$ & $\begin{array}{l}\text { Median } \\
\text { OOPE in } \\
\text { INR (IQR) }\end{array}$ & $\%$ Borrowing & $\begin{array}{l}\% \text { JSY } \\
\text { beneficiaries }\end{array}$ & \\
\hline Caste & $p<0.01$ & - & $p<0.04$ & $p=0.35$ & $p<0.01$ & - & $p<0.01$ & $p=0.11$ & $p<0.01$ & - & $p<0.02$ & $p=0.69$ & - \\
\hline Scheduled caste & $\begin{array}{l}1493 \\
(2045)\end{array}$ & $\begin{array}{l}900 \\
(500-1850)\end{array}$ & 41.3 & 44.7 & $\begin{array}{l}3902 \\
(4597)\end{array}$ & $\begin{array}{l}3000 \\
(1700-5000)\end{array}$ & 47.3 & 8.8 & $\begin{array}{l}526 \\
(1259)\end{array}$ & $\begin{array}{l}300 \\
(60-600)\end{array}$ & 33.4 & 3.8 & 16121 \\
\hline Scheduled tribe & $\begin{array}{l}1593 \\
(2428)\end{array}$ & $\begin{array}{l}1000 \\
(500-2000)\end{array}$ & 24.6 & 45.1 & $\begin{array}{l}3582 \\
(3642)\end{array}$ & $\begin{array}{l}2400 \\
(1300-5000)\end{array}$ & 32.6 & 12.2 & $314(669)$ & $\begin{array}{l}100 \\
(0-400)\end{array}$ & 16.0 & 4.8 & 16591 \\
\hline Other backward caste & $\begin{array}{l}1465 \\
(2189)\end{array}$ & $\begin{array}{l}1000 \\
(500-1800)\end{array}$ & 36.1 & 46.3 & $\begin{array}{l}4369 \\
(5193)\end{array}$ & $\begin{array}{l}3090 \\
(2000-5100)\end{array}$ & 37.0 & 7.2 & $493(931)$ & $\begin{array}{l}250 \\
(100-500)\end{array}$ & 28.7 & 2.5 & 32049 \\
\hline Others & $\begin{array}{l}2043 \\
(2447)\end{array}$ & $\begin{array}{l}1300 \\
(600-2500)\end{array}$ & 26.1 & 34.7 & $\begin{array}{l}4958 \\
(5268)\end{array}$ & $\begin{array}{l}3500 \\
(2050-6000)\end{array}$ & 22.4 & 3.2 & $553(980)$ & $\begin{array}{l}300 \\
(44-600)\end{array}$ & 20.0 & 2.4 & 16541 \\
\hline Education & $p<0.01$ & - & $p<0.01$ & $p<0.06$ & $p<0.01$ & - & $p<0.01$ & $p=0.84$ & $p<0.01$ & - & $p<0.01$ & $p=0.88$ & - \\
\hline No schooling & $\begin{array}{l}1311 \\
(1595)\end{array}$ & $\begin{array}{l}850 \\
(500-1550)\end{array}$ & 43.3 & 50.5 & $\begin{array}{l}3204 \\
(3644)\end{array}$ & $\begin{array}{l}2200 \\
(1300-3800)\end{array}$ & 53.1 & 6.4 & $408(942)$ & $\begin{array}{l}200 \\
(0-500)\end{array}$ & 29.3 & 2.5 & 39087 \\
\hline $1-5$ years & $\begin{array}{l}1494 \\
(2280)\end{array}$ & $\begin{array}{l}950 \\
(500-1700)\end{array}$ & 37.7 & 44.8 & $\begin{array}{l}3660 \\
(4078)\end{array}$ & $\begin{array}{l}2800 \\
(1600-4417)\end{array}$ & 44.0 & 7.7 & $581(997)$ & $\begin{array}{l}200 \\
(0-500)\end{array}$ & 23.6 & 4.5 & 12975 \\
\hline $6-11$ years & $\begin{array}{l}1813 \\
(2601)\end{array}$ & $\begin{array}{l}1100 \\
(530-2200)\end{array}$ & 26.7 & 38.8 & $\begin{array}{l}4613 \\
(5306)\end{array}$ & $\begin{array}{l}3200 \\
(2030-5300)\end{array}$ & 30.9 & 7.2 & $\begin{array}{l}757 \\
(1156)\end{array}$ & $\begin{array}{l}300 \\
(0-600)\end{array}$ & 17.0 & 4.6 & 24455 \\
\hline$\geq 12$ years & $\begin{array}{l}2241 \\
(2736)\end{array}$ & $\begin{array}{l}1500 \\
(600-3000)\end{array}$ & 13.4 & 32.6 & $\begin{array}{l}5727 \\
(5817)\end{array}$ & $\begin{array}{l}4250 \\
(2500-7000)\end{array}$ & 14.1 & 4.5 & $408(942)$ & $\begin{array}{l}500 \\
(100-1000)\end{array}$ & 10.4 & 4.1 & 6523 \\
\hline Wealth Index & $p<0.01$ & - & $p<0.01$ & $p<0.01$ & $p<0.01$ & - & $p<0.01$ & $p=0.45$ & $p<0.01$ & - & $p<0.02$ & $p=0.99$ & - \\
\hline Poorest & $\begin{array}{l}1240 \\
(1502)\end{array}$ & $\begin{array}{l}800 \\
(500-1500)\end{array}$ & 53.0 & 56.8 & $\begin{array}{l}2877 \\
(2837)\end{array}$ & $\begin{array}{l}2050 \\
(1100-3300)\end{array}$ & 64.2 & 8.0 & 353 (773) & $\begin{array}{l}200 \\
(0-500)\end{array}$ & 31.6 & 3.3 & 18418 \\
\hline Second & $\begin{array}{l}1417 \\
(1955)\end{array}$ & $\begin{array}{l}900 \\
(500-1600)\end{array}$ & 45.5 & 52.7 & $\begin{array}{l}3086 \\
(3428)\end{array}$ & $\begin{array}{l}2200 \\
(1200-3900)\end{array}$ & 54.9 & 8.7 & $425(780)$ & $\begin{array}{l}200 \\
(0-500)\end{array}$ & 27.9 & 3.5 & 18909 \\
\hline Middle & $\begin{array}{l}1534 \\
(1905)\end{array}$ & $\begin{array}{l}1000 \\
(500-2000)\end{array}$ & 33.9 & 43.4 & $\begin{array}{l}3701 \\
(4570)\end{array}$ & $\begin{array}{l}2600 \\
(1544-4500)\end{array}$ & 47.7 & 9.4 & $\begin{array}{l}496 \\
(1275)\end{array}$ & $\begin{array}{l}250 \\
(0-500)\end{array}$ & 21.8 & 3.4 & 17841 \\
\hline Fourth & $\begin{array}{l}1792 \\
(2680)\end{array}$ & $\begin{array}{l}1100 \\
(530-2150)\end{array}$ & 23.5 & 37.2 & $\begin{array}{l}4211 \\
(4954)\end{array}$ & $\begin{array}{l}3040 \\
(2000-5050)\end{array}$ & 36.0 & 8.2 & 596 (954) & $\begin{array}{l}300 \\
(100-700)\end{array}$ & 17.9 & 3.1 & 16171 \\
\hline Richest & $\begin{array}{l}2162 \\
(2926)\end{array}$ & $\begin{array}{l}1200 \\
(550-2800)\end{array}$ & 11.7 & 27.9 & $\begin{array}{l}5523 \\
(5692)\end{array}$ & $\begin{array}{l}4070 \\
(2500-6300)\end{array}$ & 16.0 & 3.2 & $\begin{array}{l}789 \\
(1278)\end{array}$ & $\begin{array}{l}500 \\
(200-1000)\end{array}$ & 12.7 & 2.5 & 11690 \\
\hline Area & $p=0.98$ & - & $p<0.05$ & $p<0.09$ & $p<0.01$ & - & $p<0.01$ & $p=0.55$ & $p<0.01$ & - & $p=0.87$ & $p=0.65$ & - \\
\hline Rural & $\begin{array}{l}1624 \\
(2258)\end{array}$ & $\begin{array}{l}1000 \\
(520-2000)\end{array}$ & 35.8 & 45.7 & $\begin{array}{l}4152 \\
(4472)\end{array}$ & $\begin{array}{l}3030 \\
(1800-5100)\end{array}$ & 38.5 & 7.2 & $445(860)$ & $\begin{array}{l}200 \\
(0-500)\end{array}$ & 25.3 & 3.4 & 69715 \\
\hline Urban & $\begin{array}{l}1623 \\
(2332)\end{array}$ & $\begin{array}{l}1000 \\
(468-2015)\end{array}$ & 22.5 & 34.2 & $\begin{array}{l}5112 \\
(6106)\end{array}$ & $\begin{array}{l}3500 \\
(2020-6000)\end{array}$ & 22.2 & 4.9 & $\begin{array}{l}680 \\
(1720)\end{array}$ & $\begin{array}{l}494 \\
(150-800)\end{array}$ & 24.1 & 2.3 & 13324 \\
\hline
\end{tabular}


Table 4 Mean \& median out-of-pocket-expenditure (OOPE) of normal deliveries, percent borrowings and percent JSY-beneficiaries according to socio economic profile of women (Continued)

\begin{tabular}{|c|c|c|c|c|c|c|c|c|c|c|c|c|c|}
\hline Full ANC & $p<0.01$ & - & $p=0.22$ & $p<0.07$ & $p<0.01$ & - & $p=0.09$ & $p=0.58$ & $p<0.01$ & - & $p=0.31$ & $p=0.12$ & - \\
\hline Yes & $\begin{array}{l}1862 \\
(2473)\end{array}$ & $\begin{array}{l}1100 \\
(500-2300)\end{array}$ & 26.5 & 32.8 & $\begin{array}{l}5340 \\
(5220)\end{array}$ & $\begin{array}{l}4020 \\
(2300-6200)\end{array}$ & 26.1 & 5.7 & $\begin{array}{l}707 \\
(1277)\end{array}$ & $\begin{array}{l}400 \\
(0-1000)\end{array}$ & 19.6 & 8.4 & 12010 \\
\hline No & $\begin{array}{l}1561 \\
(2213)\end{array}$ & $\begin{array}{l}1000 \\
(500-2000)\end{array}$ & 34.6 & 46.0 & $\begin{array}{l}4054 \\
(4949)\end{array}$ & $\begin{array}{l}3000 \\
(1700-5000)\end{array}$ & 36.5 & 7.9 & 449 (943) & $\begin{array}{l}200 \\
(0-500)\end{array}$ & 25.6 & 2.9 & 71013 \\
\hline $\begin{array}{l}\text { Registered } \\
\text { pregnancy and Got } \\
\text { advice on } \\
\text { institutional delivery }\end{array}$ & $p<0.01$ & - & $p=0.14$ & $p=0.25$ & $p<0.01$ & - & $p<0.06$ & $p=0.35$ & $p<0.01$ & - & $p=0.51$ & $p=0.15$ & - \\
\hline Yes & $\begin{array}{l}1767 \\
(2504)\end{array}$ & $\begin{array}{l}1050 \\
(500-2100)\end{array}$ & 29.2 & 40.1 & $\begin{array}{l}4958 \\
(5385)\end{array}$ & $\begin{array}{l}3525 \\
(2100-5748)\end{array}$ & 28.8 & 7.4 & $\begin{array}{l}590 \\
(1087)\end{array}$ & $\begin{array}{l}300 \\
(0-600)\end{array}$ & 21.9 & 6.2 & 34806 \\
\hline No & $\begin{array}{l}1413 \\
(1861)\end{array}$ & $\begin{array}{l}900 \\
(500-1600)\end{array}$ & 38.5 & 47.8 & $\begin{array}{l}3441 \\
(4183)\end{array}$ & $\begin{array}{l}2300 \\
(1400-4100)\end{array}$ & 42.1 & 4.4 & $421(922)$ & $\begin{array}{l}200 \\
(0-500)\end{array}$ & 26.4 & 2.2 & 48235 \\
\hline
\end{tabular}

INR: Indian National Rupees; SD: Standard Deviation; Borrowings: Households who borrowed money/sold property for meeting delivery expenses. 
Table 5 Mean \& median out-of-pocket-expenditure (OOPE) of caesarean-section deliveries, percent borrowings and percent JSY-beneficiaries according to socio economic profile of women

\begin{tabular}{|c|c|c|c|c|c|c|c|c|c|}
\hline \multirow{2}{*}{$\begin{array}{l}\text { Socio-demographic } \\
\text { characteristics }\end{array}$} & \multicolumn{4}{|c|}{ C-section at public institution } & \multicolumn{4}{|c|}{ C-section at private institution } & \multirow[t]{2}{*}{ Total women } \\
\hline & $\begin{array}{l}\text { Mean OOPE in } \\
\text { INR (SD) }\end{array}$ & $\begin{array}{l}\text { Median OOPE in } \\
\text { INR (IQR) }\end{array}$ & \% Borrowing & $\begin{array}{l}\% \text { JSY } \\
\text { beneficiaries }\end{array}$ & $\begin{array}{l}\text { Mean OOPE in } \\
\text { INR (SD) }\end{array}$ & $\begin{array}{l}\text { Median OOPE in } \\
\text { INR (IQR) }\end{array}$ & $\%$ Borrowing & $\begin{array}{l}\% \text { JSY } \\
\text { beneficiaries }\end{array}$ & \\
\hline Caste & $p<0.01$ & - & $p<0.01$ & $p=0.11$ & $p<0.01$ & - & $p<0.01$ & $p=0.29$ & - \\
\hline Scheduled caste & $5335(5635)$ & $3294(1500-8000)$ & 53.1 & 35.2 & $12928(8904)$ & $11000(7772-16064)$ & 63.8 & 10.1 & 1205 \\
\hline Scheduled tribe & $6060(7519)$ & $4000(1627-8000)$ & 32.5 & 30.9 & $15380(14540)$ & $12000(7100-20000)$ & 46.0 & 10.7 & 674 \\
\hline Other backward caste & $5666(6932)$ & $3400(1203-7497)$ & 44.8 & 32.7 & $13764(8320)$ & $12600(8700-18050)$ & 53.5 & 9.4 & 3044 \\
\hline Others & $6630(7321)$ & $5000(2100-9300)$ & 33.2 & 19.8 & $15220(10408)$ & 15000 (9003-20050) & 31.6 & 3.9 & 2481 \\
\hline Education & $p<0.01$ & - & $p<0.01$ & $p=0.17$ & $p<0.01$ & - & $p<0.01$ & $p=0.44$ & - \\
\hline No schooling & $5506(6396)$ & $3200(1300-7537)$ & 56.2 & 28.0 & $11675(8146)$ & $10294(5050-15500)$ & 73.5 & 7.3 & 1338 \\
\hline $1-5$ years & $4794(5633)$ & $3086(1150-6012)$ & 55.3 & 35.8 & $12206(9358)$ & 10300 (5400-15472) & 68.9 & 10.6 & 785 \\
\hline $6-11$ years & $5890(6767)$ & $4150(1700-8038)$ & 39.7 & 30.8 & 14013 (9172) & 12700 (8816-18300) & 48.9 & 8.9 & 3249 \\
\hline$\geq 12$ years & 7197 (8043) & $5023(2150-10198)$ & 21.1 & 22.2 & 16118 (10374) & $15013(10020-20100)$ & 27.8 & 5.3 & 2180 \\
\hline Wealth Index & $p<0.01$ & - & $p<0.01$ & $p<0.01$ & $p<0.01$ & - & $p<0.01$ & $p=0.13$ & - \\
\hline Poorest & 5326 (7075) & $2604(1200-6756)$ & 70.1 & 39.9 & $12363(8205)$ & $10600(5042-17187)$ & 82.8 & 14.8 & 401 \\
\hline Second & $5304(5803)$ & $3452(1500-7100)$ & 63.7 & 39.3 & $12593(8333)$ & 10599 (7008-16057) & 76.7 & 11.5 & 722 \\
\hline Middle & $4926(5038)$ & $3267(1300-7135)$ & 54.2 & 38.4 & $12397(8672)$ & 10700 (6400-15500) & 73.1 & 11.8 & 1211 \\
\hline Fourth & $6247(7306)$ & $4726(2000-8200)$ & 40.7 & 28.0 & $13074(8176)$ & $12050(7404-17000)$ & 56.8 & 9.9 & 1997 \\
\hline Richest & $6709(7684)$ & $5000(2022-9247)$ & 18.8 & 18.2 & $15723(10546)$ & 15000 (10000-20070) & 28.6 & 4.3 & 3217 \\
\hline Area & $p=0.37$ & - & $p<0.03$ & $p=0.21$ & $p<0.01$ & - & $p<0.01$ & $p=0.42$ & - \\
\hline Rural & $5850(6537)$ & $4000(1700-4000)$ & 46.2 & 31.9 & $13549(8808)$ & $12300(8000-18000)$ & 55.8 & 8.8 & 4831 \\
\hline Urban & $6117(7497)$ & $4400(1510-8100)$ & 30.8 & 23.5 & $15422(10723)$ & $15000(10000-20020)$ & 32.6 & 5.6 & 2721 \\
\hline Full ANC & $p=0.65$ & - & $p=0.77$ & $p=0.88$ & $p<0.01$ & - & $p=0.16$ & $p=0.55$ & - \\
\hline Yes & 5851 (6292) & $4200(1700-8000)$ & 40.4 & 30.3 & $15233(9563)$ & 15000 (10000-20010) & 41.2 & 9.4 & 2997 \\
\hline No & $5982(7178)$ & $4000(1510-8187)$ & 41.8 & 28.5 & 13539 (9635) & $12100(7200-18050)$ & 50.8 & 6.1 & 4548 \\
\hline $\begin{array}{l}\text { Registered pregnancy } \\
\text { and Got advice on } \\
\text { institutional delivery }\end{array}$ & $p<0.07$ & - & $p=0.15$ & $p=0.88$ & $p<0.01$ & - & $p=0.10$ & $p=0.15$ & - \\
\hline Yes & $5805(6280)$ & $4050(1700-8042)$ & 39.1 & 29.1 & 14717 (9712) & 13831 (9578-20000) & 44.2 & 8.6 & 5822 \\
\hline No & $6441(8741)$ & $4005(1500-8400)$ & 49.4 & 29.6 & 12605 (9173) & $10623(5500-16257)$ & 55.5 & 3.7 & 1729 \\
\hline
\end{tabular}

C-section: Caesarean-section; INR: Indian National Rupees; SD: Standard Deviation; Borrowings: Households who borrowed money/sold property for meeting delivery expenses. 
transfer within this programme. Even among women who had normal deliveries in public institutions, JSY use was less than $50 \%$ in 29 of the 34 states/UTs in India, highlighting scope for further improvement. Increased literacy and wealth were associated with a higher likelihood of an institutional delivery, but higher OOPE and no major variations in use of the JSY programme.

\section{How comparable are our results with other studies?}

The Coverage Evaluation Survey (CES-2009) [12] report estimated mean expenditure for transporting a pregnant woman to facility in India at Rs. 192, while it was Rs. 322 in our study. A study done [25] in 12 districts of Uttar Pradesh reported average expenditure for institutional deliveries to be Rs. 1,179, which closely matches with our estimate for Uttar Pradesh (Rs. 1,246). Another cross-sectional survey from 12 districts of eight high focus - non NE states [26] (excluding Jammu \& Kashmir and Himachal Pradesh) in 2010, reported average expenditure of an institutional delivery (excluding transportation) to be Rs. 1,028 , while our mean expenditure of a normal institutional delivery in these eight states was Rs. 1,719 ( $\mathrm{SD}=1,924)$. These variations in expenditures may be due to variations in the percentage of private hospital deliveries, $11 \%$ in our study and $5 \%$ in the reported study [26].

A comparison of our results (based on 2007/08 data) with those from the National Sample Survey Organization (NSSO) conducted in 2004 [27] suggests that OOPE to families for public and private institution delivery may have increased during this time period. In 2004, OOPE on a public, private and a home delivery respectively was Rs. 1,387, Rs. 6,094, and Rs. 428; while OOPEs in 2007/08 were Rs. 2,103, Rs. 7,245 and Rs. 466 respectively. There was no major increase in expenditure on home deliveries over this period. This data suggests that the JSY programme may not have offset increases in OOPE over that time period for many families.

Our findings suggest that the proportion of women opting for home deliveries in 2007/08 remains high (52\%) in India; although a more recent (2009/10) estimate [12] found it to be $27 \%$, suggesting that the JSY programme may have been successful in reducing the proportion of home deliveries since the DLHS-3 (2007-08) was conducted. Women from high focus-non NE states (where substantial portion of deliveries were at home) cited the following reasons for opting 'home as the place of delivery' in their previous pregnancy: not necessary to go to institution (33\%); cost of institutional delivery was too much (25\%); no time to go to institution (24\%); better care at home (17\%); institution too far/no transport (12\%); lack of knowledge (7\%); family did not allow (7\%); not customary (7\%); poor quality of service at institution (5\%). This implies that barriers other than OOPE, including availability, accessibility, and lack of planning and cultural reasons need to be addressed to reduce home deliveries in India.

A cross-sectional survey [28] in 2008 found that the average amount paid by JSY beneficiaries to an institution for medicines and other services ranged from Rs. 299 in Madhya Pradesh to Rs. 1,638 in Orissa. These findings are consistent with ours, and imply that the JSY benefit is insufficient to cover expenditures incurred on delivery, thus, requiring many families to borrow money to pay for this. This is confirmed by our finding that rural families from high focus- non NE states had average additional expenditures of Rs. 544 and Rs. 4,761 for public and private institution deliveries respectively after receiving the JSY benefit (Rs 1400). Further, mean OOPE to families for normal deliveries in public institutions was more than the JSY-compensation amount of (Rs. $1,400)$ in five of the 10 high focus- non NE states.

\section{Study strengths and limitations}

This study provides some of the first robust state-level estimates of OOPE for normal and c-section deliveries, the proportion of families required to borrow to meet these expenditures and the reach of JSY-programme, by location of delivery in India. One of the limitations of our study is OOPE to family on delivery care reported here are based on the figures recalled by women. Studies that gather expenditures of families from hospital records [4] are often more accurate as they are not influenced by recall or reporting bias. The current study only included direct expenses such as transportation and facility-based expenses. It did not include indirect expenses such as spending by women and families on food, other purchases during hospitalization/delivery, wages lost by women and family members during the delivery process and bribes/gifts. Results of this study must be seen in the light of limitations of the methods of DLHS-3 [29] which did not capture the reasons for variable implementation and use of JSY between different states [14], including eligibility guidelines, awareness of JSY programme, amount distributed, payment process, delays in payments to mothers and involvement of Associated Social Health Activists (ASHAs) in maternity care[25-27]. Before streamlining of JSY programme in 2007-08, there was very little change in the distribution of institutional deliveries during 2002-04 [30] and 2005-06 [11]. In 2009 proportion of institutional deliveries in India increased to $73 \%$ and JSY use increased to $33 \%$ [12], clearly implying that the coverage of the JSY has increased since 2007-08, and our findings are unlikely to reflect current JSY use and distribution of location of delivery, even though OOPE and family borrowings may not have changed markedly since 2007-08. Hence, ongoing evaluation of the JSY programme is essential to establish 
whether its reach and impacts on OOPE and family borrowings have improved.

\section{Policy implications}

Our results highlight the ongoing high OOPE of Indian families for delivery/maternity care, resulting in $25-47 \%$ families in India having to borrow money to meet pregnancy/delivery related expenses. The OOPE burden was found to be especially high in: low wealth index, illiterate/ less educated and low social group families and low percapita income states [31]. The high levels of OOPE found, low reported use of the JSY programme and given that expenditures exceed the financial benefit of this programme for many families, suggest that the impact of programme on OOPE in 2007/08 appears to have been modest. Additional investment in the JSY programme, strengthening state-specific interventions targeting population groups most likely to avoid institutional care due to OOPE and providing support to families in financial planning for maternity care are likely to be required in order to meet the MDGs 4 and 5 in India.

\section{Conclusions}

Our study highlights the ongoing high OOPE and impoverishing impact of institutional delivery care in India despite a high profile policy initiative seeking to address this issue. Additional investment in JSY and strengthening of state level implementation is required to increase coverage of JSY programme, reduce maternity related OOPE, reduce delivery associated borrowings and increase the proportion of institutional deliveries in India. Such an investment is vital to accelerate progress towards achievement of MDGs 4 and 5.

\section{Endnotes}

${ }^{\mathrm{a}} 12$ cases out of 36,536 were excluded from analysis as outliers

${ }^{b} 14$ cases out of 83,524 were excluded from analysis as outliers

\section{Abbreviations \\ ANC: Antenatal care; ANOVA: Analysis of variance; ASHA: Accredited Social, Health Activist; AYUSH: Ayurveda, Yoga, Unani, Siddha \& Homeopathy; BPL: Below Poverty Line; CES: Coverage Evaluation Survey; C- section: Caesarean section; DLHS: District Level Household and facility Survey; GBP: British Pound sterling; INR: Indian Rupee; IQR: Inter-quartile range; JSY: Janani Suraksha Yojana; MDG: Millennium Development Goals; MMR: Maternal Mortality Ratio; NE: North Eastern; NGO: Non-governmental organisation; NRHM: National Rural Health Mission; NSSO: National Sample Survey Organisation; OOPE: Out of pocket expenditure; SD: Standard deviation; SPSS: Statistical Package for Social Sciences; U5MR: Under five mortality rate; UK: United Kingdom; UT: Union-Territory.}

\section{Competing interests}

The authors have no financial benefits or competing interests related to this work.

\section{Author's contributions}

HRM led conceptualization, literature review, conducted all analysis, and led manuscript development and finalization. MK assisted with literature review. AK assisted with conceptualization and analysis. CM assisted with analytic approach, literature review, and editing of the manuscript. All the authors participated as described above and all read and approved this final submitted manuscript.

\section{Acknowledgement}

This research was supported by a Wellcome Trust Capacity Strengthening Strategic Award to the Public Health Foundation of India and a consortium of UK universities. We thank Prof Shah Ebrahim for providing valuable inputs during different stages of manuscript development and Dr Fiona Taylor for the encouragement to develop this paper. We are also thankful to Dr Sukumar Vellakkal, Dr Anil Chandran, Dr Manas R Pradahan and Mr Dilip for reviewing the manuscript and making edits. Thanks also to Aditi Bam, Aishwarya Pillai and Dr Jai for editing, Geeta Nambiar for developing Endnote file and to Professor Kulkarni for reviewing the paper and providing helpful inputs. CM is funded by the Higher Education Funding Council for England and the National Institute for Health Research.

\section{Author details}

${ }^{1}$ South Asia Network for Chronic Disease (SANCD), Public Health Foundation of India (PHFI), C1/52, First Floor, Safdarjung Development Area, New Delhi 110 016, India. ${ }^{2}$ The Vistaar Project, IntraHealth International Inc., New Delhi, India. ${ }^{3}$ The Vistaar Project, IntraHealth International, Inc., Ranchi, Jharkhand, India. ${ }^{4}$ School of Public Health, Imperial College, London, United Kingdom.

Received: 17 May 2012 Accepted: 24 November 2012

Published: 5 December 2012

\section{References}

1. Kesterton AJ, et al: Institutional delivery in rural India: the relative importance of accessibility and economic status. BMC Pregnancy Childbirth 2010, 10:30.

2. Nalin T: Paying the price: Institutional delivery costs keep pregnant women at home; 2011. Available from: [http://governancenow.com/views/think-tanks/ paying-price-institutional-delivery-costs-keep-pregnant-women-home]

3. National Commission on Macroeconomics and Health, Report of the National Commission on Microeconomics and Health: Ministry of Health and Family Welfare. New Delhi: Government of India; 2005.

4. Borghi J, Storeng K, Filippi V: Overview of the costs of obstetric care and the economic and social consequences for households. Stud Health Serv Organ Policy 2008, 24:27-52.

5. Balarajan Y, Selvaraj S, Subramanian SV: Health care and equity in India. Lancet 2011, 377(9764):505-15.

6. Selvaraj S, Karan AK: Deepening health insecurity in India: evidence from national sample surveys since 1980s. Econ Pol Wkly 2009, 44:55-60

7. FHI: Skilled care during child birth - Policy Brief. 588 Broadway, Suit 503, New York: Family Care International Inc; 2002.

8. Office of the Registrar General, G.o.l: Sample Registration System. Maternal Mortality in India 1997-2003, Trends, Causes and Risk Factors; 2008.

9. Office of the Registrar General, G.o.l: Sample Registration System. Special bulletin on Maternal Mortality in India 2007-09; 2011.

10. IIPS: National family Health Survey (MCH and Family Planning), India 1992-93. Bombay: International Institute for Population Sciences; 1995.

11. IIPS: National Family Health Survey (NFHS-3), 2005-06: India. Mumbai, India: International Institute for Population Sciences and Macro International; 2007.

12. UNICEF: Coverage Evaluation Survey (CES-2009). New Delhi: All India Report; 2009.

13. Paul VK, et al: Reproductive health, and child health and nutrition in India: meeting the challenge. Lancet 2011, 377(9762):332-349.

14. Lim SS, et al: India's Janani Suraksha Yojana, a conditional cash transfer programme to increase births in health facilities: an impact evaluation. Lancet 2010, 375(9730):2009-23.

15. Paul VK: India: conditional cash transfers for in-facility deliveries. Lancet 2010, 375(9730):1943-4.

16. Annual report to the people on health. New Delhi: Ministry of Health and Family Welfare, Government of India; 2010.

17. Janani Suraksha Yojana: Guidelines for Implementation. New Delhi: Ministry of Health and Family Welfare, Govt. of India. 
18. Vyas $\mathrm{S}$, et al: A cost analysis of deliveries conducted in various health care settings in a city of India. Healthline 2011, 1(1):61-64.

19. Satapathy DM, Panda R, Das BC: Cost factors related with normal vaginal delivery and caesarean section in government and non-government settings. Indian J Community Medicine 2005, 30(2):66-67.

20. Rajeswari B, Dilip TR, Duggal R: Utilization of and expenditure on delivery care services: Some observations from Nasik district, Maharashtra, Volume 7(2). New Delhi, India: Regional health forum WHO South-East Asia region; 2003:34-41.

21. lyengar SD, et al: Comparison of domiciliary and institutional deliverycare practices in rural Rajasthan, India. J Health Popul Nutr 2009, 27(2):303-12.

22. Hotchkiss DR, Kanjilal B, Sharma S, Sodani PR, Chakraborty G: Household expenditure on reproductive and child health care services in Udaipur, Rajasthan. In Financing reproductive and child health care in Rajasthan. New Delhi, India: The Futures Group International, POLICY Project; and the Indian Institute of Health Management Research; 2000.

23. IIPS and G.o.I. Ministry of Health and Family Welfare: District Level Household Survey (DLHS-3). Interviewer's Manual. Mumbai: International Institute for Population Sciences; 2007-08.

24. NRHM Health Management Information System (HMIS) Portal; 2012. Available from: [http://www.nrhm-mis.nic.in/PublicPeriodicNRHMReports.aspx]

25. Khan ME, Hazra A, Bhatnagar I: Impact of JSY on selected family health behaviors in rural Uttar Pradesh. J Fam Welf 2010, 56:9-22.

26. Programme evaluation of the Janani Suraksha Yojana. New Delhi: National Health System Resource Centre, Ministry of Health \& Family Welfare, Government of India; 2011.

27. Bonu S, Bhushan I, Rani M, Anderson I: Incidence and correlates of 'catastrophic' maternal health care expenditure in India. Health Policy Plan 2009, 24(6):445-456

28. Concurrent Assessment of Janani Suraksha Yojana scheme in selected states of India, 2008. New Delhi: UNFPA; 2009.

29. IIPS: District Level Household and Facility Survey (DLHS-3), 2007-08; India. Mumbai: International Institute for Population Sciences; 2010.

30. Economic survey 2011-12: Statistical appendix. New Delhi: Ministry of Finance, Government of India.

31. Ministry of Finance, G.o.l: Economic survey 2011-12: Statistical appendix New Delhi.

doi:10.1186/1471-2458-12-1048

Cite this article as: Modugu et al:: State and socio-demographic group variation in out-of-pocket expenditure, borrowings and Janani Suraksha Yojana (JSY) programme use for birth deliveries in India. BMC Public Health 2012 12:1048.

\section{Submit your next manuscript to BioMed Central and take full advantage of:}

- Convenient online submission

- Thorough peer review

- No space constraints or color figure charges

- Immediate publication on acceptance

- Inclusion in PubMed, CAS, Scopus and Google Scholar

- Research which is freely available for redistribution 